\title{
QRD-Based Antenna Selection for ML Detection of Spatial Multiplexing MIMO Systems: Algorithms and Applications
}

\author{
Chun-Tao Lin, Student Member, IEEE, and Wen-Rong Wu, Member, IEEE
}

\begin{abstract}
Antenna selection is a simple but effective technique to enhance the performance of a spatial multiplexing multipleinput-multiple-output (MIMO) system. The selection criterion depends on the detector used at the receiver. For the maximumlikelihood detector, the criterion is to maximize the free distance. However, an exhaustive search is required to derive the distance, and the computational complexity can be prohibitively high. To avoid the exhaustive search, a lower bound of the free distance derived with the singular value decomposition (SVD) was then developed. This bound only involves the smallest singular value of the channel matrix, and its maximization can easily be conducted. An alternative lower bound of the free distance with the QR decomposition (QRD) was also derived in the literature. In this paper, we first propose a QRD-based selection method maximizing the lower bound. With some matrix properties, we theoretically prove that the lower bound yielded by the QRD is tighter than that by the SVD. We then propose a basis transformation method so that the lower bound yielded by the QRD can further be tightened. As a result, the QRD-based selection method can achieve near optimum performance. Finally, we extend the use of the proposed methods to other applications, such as receive antenna selection, joint transmit/receive antenna selection, and antenna selection in MIMO relay systems. Simulations show that the proposed selection methods can significantly outperform existing methods.
\end{abstract}

Index Terms-Antenna selection, free distance, multipleinput-multiple-output (MIMO), QR decomposition (QRD), spatial multiplexing.

\section{INTRODUCTION}

\section{A. Background}

M ULTIPLE-input-multiple-output (MIMO) technology has been widely adopted in wireless communication systems since it can provide higher spectral efficiency without bandwidth expansion [1]. Consider a MIMO system with $N_{t}$ transmit antennas and $N_{r}$ receive antennas. If we let $N_{t}=M$ and $N_{r}=M$, then $M$ independent bit streams can simultaneously be transmitted. This scheme is generally referred to as spatial multiplexing. However, the performance of spatial multiplexing can seriously be affected in fading environments

Manuscript received September 17, 2010; revised February 21, 2011 and April 22, 2011; accepted May 27, 2011. Date of publication June 20, 2011; date of current version September 19,2011. This work was supported in part by the National Science Council under Grant NSC-98-2221-E-009-046-MY3 and was presented at the 20th Annual IEEE International Symposium on Personal, Indoor, and Mobile Radio Communications, Tokyo, Japan, September 2009. The review of this paper was coordinated by Prof. G. Bauch.

The authors are with the Institute of Communications Engineering, National Chiao Tung University, Hsinchu 300, Taiwan (e-mail: tow.cm91g@ nctu.edu.tw;wrwu@faculty.nctu.edu.tw).

Digital Object Identifier 10.1109/TVT.2011.2160003
[2]. One way to combat the fading is to let $N_{t}>M$ and $N_{r}>$ $M$ so that higher spatial diversity can be achieved. The main disadvantage of this approach is that the implementation cost can be much higher than that of the original system. Transmit antenna selection is one effective yet simple solution to solve the problem. The idea of this scheme comes from the fact that the cost of antennas is low, whereas that of radio-frequency (RF) chains is relatively high. In transmit antenna selection, only $M$ antennas are selected for signal transmission, although there are $N_{t}$ antennas. With a low-rate feedback channel, the transmitter can conduct optimum selection such that the performance of the $M \times N_{r}$ system can approach that of the unselected $N_{t} \times N_{r}$ system. Since the number of RF chains is reduced, the implementation cost of the MIMO system can be reduced. In this paper, we investigate the selection methods for spatial multiplexing MIMO systems with maximum-likelihood (ML) detection.

\section{B. Related Work}

The transmit antenna selection criterion design for a spatial multiplexing MIMO system has extensively been studied. In [3], the performance of antenna selection using the capacity maximization criterion was theoretically analyzed. The capacity-based method is derived from a general formula of the MIMO channel capacity, which is independent of the receiver type. Therefore, maximizing the channel capacity may not provide the optimum performance in some receivers. In [4], several selection criteria for linear receivers have been proposed, including post signal-to-noise ratio (SNR) maximization and mean-square-error minimization. In [5], the selection method aiming to optimize the performance of ordered successive interference cancellation was proposed. For the ML detector, the general criterion is to minimize the error probability. In [6], a selection method minimizing the union upper bound of the error probability was derived; however, the computational complexity of this method can be very high. It is well known that the error probability of the ML detection under high SNR is determined by the minimum Euclidean distance between the received signal constellation, which is generally referred to as free distance. To have the optimum performance, we can then maximize the free distance in the selection problem [7]. Unfortunately, finding the free distance requires an exhaustive search, and the computational complexity can be prohibitively high. To reduce the computational complexity, a method utilizing the singular value decomposition (SVD) was proposed in [7]. The 
SVD-based method selects the antenna subset that maximizes the smallest singular value of the channel matrix. It was shown that this singular value can serve as a lower bound of the free distance. Although the computational complexity of the SVDbased method is low, the solution is suboptimum. In addition, the tightness of the lower bound has not been analyzed.

\section{Contributions of Our Work}

In this paper, we develop new methods providing tight lower bounds in the antenna selection problem. We first propose a QR decomposition (QRD)-based selection method maximizing the smallest diagonal entry in the R-factor of the channel matrix, where the R-factor is the upper triangular matrix obtained with the QRD of the channel matrix. We theoretically prove that the lower bound achieved with the QRD-based method is tighter than that with the SVD-based method. The tightness of the lower bound is related to the spread of the diagonal entries in the $\mathrm{R}$-factor. If the spread is smaller, then the lower bound is tighter. This property motivates us to propose a basis transformation method, further tightening the lower bound of the QRD-based method. The idea is to find a basis transformation for the transmitted symbol vector so that the spread can be reduced. In this paper, we propose two basis transformation matrices to do the work. The first matrix is the permutation matrix, and the second matrix is the transformation matrix used in lattice reduction (LR) [8], [9]. The LR technique has been used in antenna selection [10] for performance improvement. The selection method in [10] is designed for a linear detector operated in the basis-transformed domain. In this paper, LR is used only for the derivation of the transformation matrix. An ML detector operating in the original basis is used at the receiver. Therefore, the role of the LR is much different from that in [10]. We theoretically prove that the proposed basis transformations can further tighten the lower bound obtained with the original QRD-based method. The basis transformation method needs extra QRDs, and the computational complexity will be increased. We propose an efficient permutation method and the use of Givens rotations to reduce the computational complexity of our selection methods.

Except for transmit antenna selection, we also consider the applications of the proposed algorithms in other scenarios, which include receive antenna selection [11]-[13], joint transmit/receive antenna selection [14], and antenna selection in MIMO relay systems. For receive antenna selection, no feedback is required, and this will be a great advantage in high-mobility environments. In some scenarios, the number of receive antenna elements may be limited due to size constraints. Joint transmit/receive antenna selection provides a solution to this problem. MIMO relay systems have recently been extensively studied [15], [16] since they can provide range extension or diversity enhancement for MIMO systems. In general, it is desirable to minimize the hardware complexity in a relay. Antenna selection is then a good candidate for performance enhancement in the system.

The remainder of this paper is organized as follows. Section II describes the lower bounds obtained with the SVDand QRD-based methods. Section III describes the proposed

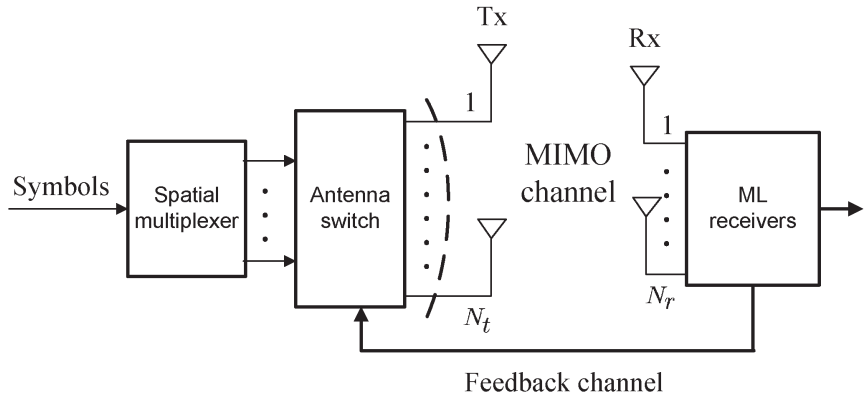

Fig. 1. System model for transmit antenna selection in a spatial multiplexing MIMO system.

basis transformation method. Section IV considers the implementation issues, and Section V describes the applications of the proposed methods. Section VI reports the simulation results evaluating the performance of our proposed algorithms. Finally, Section VII draws conclusions.

\section{LOWER BOUndS FOR FreE DistANCE}

\section{A. System and Signal Models}

Consider a spatial multiplexing wireless MIMO system with $N_{t}$ transmit antennas and $N_{r}$ receive antennas, as described in Fig. 1. Let $N_{t}>M, N_{r} \geq M$, and $\mathbf{H}$ denote the $N_{r} \times N_{t}$ channel matrix. In transmit antenna selection, the receiver first selects $M$ transmit antennas according to a selection criterion, where $M$ is the number of transmitted bit streams. Let each transmit antenna subset be represented by an index $p$. Then, via a feedback channel, the receiver sends the index of the optimum antenna subset back to the transmitter. Finally, the transmitter uses the selected antennas for signal transmission. Note that there are $\left(\begin{array}{c}N_{t} \\ M\end{array}\right)$ antenna subsets, each of which corresponds to an $N_{r} \times M$ MIMO channel. Let $x_{i}$ be the symbol transmitted at antenna $i$, and let $\mathbf{x}=\left[x_{1}, x_{2}, \ldots, x_{M}\right]^{T}$, where (.) $)^{T}$ represents the transpose operation. The corresponding received signal vector can then be expressed as

$$
\mathbf{y}=\mathbf{H}_{p} \mathbf{x}+\mathbf{n}
$$

where $\mathbf{H}_{p}$ is the channel matrix corresponding to the selected antenna subset, and $\mathbf{n}$ is the $N_{r} \times 1$ Gaussian noise vector. Assume that each entry of $\mathbf{n}$ is independent identically distributed (i.i.d.) with the covariance matrix of $\sigma^{2} \mathbf{I}_{N_{r}}$, where $\sigma^{2}$ is the noise variance, and $\mathbf{I}_{N_{r}}$ is an $N_{r} \times N_{r}$ identity matrix. The ML detector searches all the possible symbol vectors to obtain an estimate $\hat{\mathbf{x}}$ such that

$$
\hat{\mathbf{x}}=\min _{\mathbf{x} \in \mathcal{X}^{M}}\left\|\mathbf{y}-\mathbf{H}_{p} \mathbf{x}\right\|^{2}
$$

where $\mathcal{X}^{M}$ is a set consisting of all the possible transmitted symbol vectors. We also define $\mathcal{X}^{M}$ as the symbol vector constellation of $\mathbf{x}$.

It is well known that the performance of ML detection in high SNR depends on the free distance defined as

$$
\begin{aligned}
d_{\text {free }} & =\min _{\mathbf{x}, \mathbf{x}^{\prime} \in \mathcal{X}^{M}, \mathbf{x} \neq \mathbf{x}^{\prime}}\left\|\mathbf{H}_{p}\left(\mathbf{x}-\mathbf{x}^{\prime}\right)\right\|^{2} \\
& =\min _{\mathbf{x}, \mathbf{x}^{\prime} \in \mathcal{X}^{M}, \mathbf{x} \neq \mathbf{x}^{\prime}}\left(\mathbf{x}-\mathbf{x}^{\prime}\right)^{H} \mathbf{H}_{p}^{H} \mathbf{H}_{p}\left(\mathbf{x}-\mathbf{x}^{\prime}\right)
\end{aligned}
$$


where $(.)^{H}$ represents the Hermitian operation, and $\left(\mathbf{x}-\mathbf{x}^{\prime}\right)$ is the difference vector. The free distance represents the minimum distance of the received signal constellation. Therefore, the optimum antenna selection criterion [7] for the ML receiver is equivalent to choosing the antenna subset whose $\mathbf{H}_{p}$ gives the maximum free distance. We can compute the free distance of each candidate channel matrix using (3) and then choose the antenna subset with the largest $d_{\text {free }}$. This optimum solution can be found by an exhaustive search over all possible $\left(\begin{array}{l}N_{t} \\ M\end{array}\right)$ candidate channel matrices and all difference vectors. However, this exhaustive search requires very high computational complexity when considering a large number of transmitted bit streams with a large constellation modulation scheme. A suboptimum approach is considered to minimize a lower bound of the free distance instead of the free distance itself.

\section{B. Lower Bound With SVD-Based Method}

Let $\mathbf{H}_{p}$ be an $N_{r} \times M$ full column-rank matrix with its SVD given as $\mathbf{H}_{p}=\mathbf{U} \boldsymbol{\Lambda} \mathbf{V}^{H}$, where $\mathbf{U}$ is an $N_{r} \times N_{r}$ unitary matrix, $\mathbf{V}$ is an $M \times M$ unitary matrix, and $\boldsymbol{\Lambda}$ is an $N_{r} \times M$ diagonal matrix. The nonzero entries of $\Lambda$ are the singular values of $\mathbf{H}_{p}$. Define the symbol constellation of $x_{k}$ as $\mathcal{X}_{k}$ and the minimum distance of $\mathcal{X}_{k}$ as

$$
d_{\min }\left(\mathcal{X}_{k}\right)=\min _{x_{k}, x_{k}^{\prime} \in \mathcal{X}_{k}, x_{k} \neq x_{k}^{\prime}}\left|x_{k}-x_{k}^{\prime}\right|^{2} .
$$

In addition, define the minimum distance of the symbol vector constellation $\mathcal{X}^{M}$ as

$$
d_{\min }\left(\mathcal{X}^{M}\right)=\min _{\mathbf{x}, \mathbf{x}^{\prime} \in \mathcal{X}^{M}, \mathbf{x} \neq \mathbf{x}^{\prime}}\left\|\mathbf{x}-\mathbf{x}^{\prime}\right\|^{2}
$$

In a spatial multiplexing MIMO system, $x_{k}$ 's are usually uncorrelated. Thus, we can have

$$
d_{\min }\left(\mathcal{X}^{M}\right)=\min \left\{d_{\min }\left(\mathcal{X}_{1}\right), d_{\min }\left(\mathcal{X}_{2}\right), \ldots, d_{\min }\left(\mathcal{X}_{M}\right)\right\}
$$

Note that if $x_{k}$ 's are correlated, then (6) is not valid in general. With (6), $d_{\min }\left(\mathcal{X}^{M}\right)$ can easily be computed for quadraticamplitude modulation (QAM) constellations. Let a QAM symbol be represented by $a_{I}+j a_{Q}$, where $a_{I} \in\{ \pm 1, \pm 3, \cdots\}$, and $a_{Q} \in\{ \pm 1, \pm 3, \cdots\}$. We then have $d_{\min }\left(\mathcal{X}_{1}\right)=d_{\min }\left(\mathcal{X}_{2}\right)=$ $\cdots=d_{\min }\left(\mathcal{X}_{M}\right)=4$ and $d_{\min }\left(\mathcal{X}^{M}\right)=\min \{4,4, \ldots, 4\}=4$.

Using the Rayleigh-Ritz theorem, the SVD-based lower bound of the free distance was derived in [7] as

$$
d_{\text {free }} \geq \lambda_{M}^{2} d_{\min }\left(\mathcal{X}^{M}\right)
$$

where $\lambda_{M}$ is the minimum singular value of the matrix $\mathbf{H}_{p}$. Note that (7) is different from that in [7] by a factor of $M$. The reason for this is that the free distance in our application is a relative not absolute value. Since all $\mathbf{H}_{p}$ 's are of the same dimension, scaling the free distance will not change the selection result. Thus, the factor $M$ is omitted for simplicity in this paper. The lower bound in (7) indicates that the free distance can be evaluated with $\lambda_{M}$ and $d_{\min }\left(\mathcal{X}^{M}\right)$. It is simple to see that the value of $d_{\min }\left(\mathcal{X}^{M}\right)$ is the same for each $\mathbf{H}_{p}$.
Thus, with the SVD-based method, only the minimum singular value of each $\mathbf{H}_{p}$ is required to compute, and the computational complexity can be reduced dramatically. However, the main problem for the SVD-based method is that the lower bound (7) may not be tight enough. An alternative lower bound of the free distance derived from the QRD was developed in [17]. In this paper, we propose the use of this lower bound for solving the antenna selection problem.

\section{Lower Bound With QRD-Based Method}

The matrix $\mathbf{H}_{p}$ can be factorized in the form of $\mathbf{H}_{p}=\mathbf{Q R}$, where $\mathbf{Q}$ is an $N_{r} \times M$ column-wise orthonormal matrix, and $\mathbf{R}$ is an $M \times M$ upper triangular matrix with positive realvalued diagonal entries as

$$
\mathbf{R}=\left[\begin{array}{cccc}
R_{1,1} & R_{1,2} & \ldots & R_{1, M} \\
0 & R_{2,2} & \ldots & R_{2, M} \\
\vdots & \vdots & \ddots & \vdots \\
0 & 0 & \ldots & R_{M, M}
\end{array}\right]
$$

As mentioned, the matrix $\mathbf{R}$ is also referred to as the $\mathrm{R}$-factor [17] of $\mathbf{H}_{p}$. Let $[\mathbf{R}]_{k}$ denote the $k$ th diagonal entry of $\mathbf{R}$. Via this decomposition, we can have another lower bound of the free distance as

$$
\begin{aligned}
d_{\text {free }} & \geq\left(\min _{1 \leq k \leq M}[\mathbf{R}]_{k}^{2}\right) d_{\min }\left(\mathcal{X}^{M}\right) \\
& =[\mathbf{R}]_{\min }^{2} d_{\min }\left(\mathcal{X}^{M}\right)
\end{aligned}
$$

where $[\mathbf{R}]_{\min }$ represents the minimum diagonal entry in the matrix $\mathbf{R}$. Thus, we propose the use of (8) as a selection criterion, which is referred to as QRD-based method in this paper. In what follows, we show that the lower bound obtained with the QRD-based method is tighter than that with the SVDbased method.

For an arbitrary $M \times M$ positive-definite matrix $\mathbf{A}=$ $\mathbf{H}_{p}^{H} \mathbf{H}_{p}$, we can have its eigenvalue decomposition expressed as $\mathbf{A}=\mathbf{V} \boldsymbol{\Sigma} \mathbf{V}^{H}$, where $\boldsymbol{\Sigma}$ is an $M \times M$ diagonal matrix whose nonzero entries are the eigenvalues of $\mathbf{A}$. It is known that a positive-definite matrix can also be decomposed by the Cholesky factorization in the form of $\mathbf{A}=\mathbf{B D B}^{H}$, where $\mathbf{D}$ is an $M \times M$ diagonal matrix, and $\mathbf{B}$ is an $M \times M$ unit lower triangular matrix expressed as

$$
\mathbf{B}=\left[\begin{array}{cccc}
1 & 0 & \ldots & 0 \\
B_{2,1} & 1 & \ldots & 0 \\
\vdots & \vdots & \ddots & \vdots \\
B_{M, 1} & B_{M, 2} & \ldots & 1
\end{array}\right] .
$$

The diagonal entries of $\mathbf{D}$ are also referred to as Cholesky values [18]. Consider two sequences $\underline{\sigma}=\left(\sigma_{1}, \sigma_{2}, \ldots, \sigma_{M}\right)$ and $\underline{d}=\left(d_{1}, d_{2}, \ldots, d_{M}\right)$ consisting of the eigenvalues and Cholesky values of $\mathbf{A}$, respectively. Note that the entries of both sequences $\underline{\sigma}$ and $\underline{d}$ are arranged in descending order so that $\sigma_{1} \geq \sigma_{2} \geq \cdots \geq \sigma_{M}$ and $d_{1} \geq d_{2} \geq \cdots \geq d_{M}$. With the preceding definitions, we can have the following proposition. 
Proposition 1: For an $N_{r} \times M$ full column-rank matrix $\mathbf{H}_{p}$ with its QRD and SVD expressed as $\mathbf{H}_{p}=\mathbf{Q R}$ and $\mathbf{H}_{p}=$ $\mathbf{U} \boldsymbol{\Lambda} \mathbf{V}^{H}$, respectively, the inequality $\lambda_{M} \leq[\mathbf{R}]_{\text {min }}$ holds true for all channel realizations.

Proof: It is known that the Cholesky factorization of a positive-definite matrix $\mathbf{A}=\mathbf{H}_{p}^{H} \mathbf{H}_{p}$ is unique [19] and can be expressed as $\mathbf{A}=\mathbf{B D B}^{H}$. With the $\mathrm{QRD} \mathbf{H}_{p}=\mathbf{Q R}$, we can also have

$$
\mathbf{A}=\mathbf{R}^{H} \mathbf{R}=\mathbf{R}_{\mathrm{u}}^{H} \mathbf{D}^{\prime} \mathbf{R}_{\mathrm{u}}
$$

where $\mathbf{R}_{\mathrm{u}}$ is an $M \times M$ unit upper triangular matrix, and $\mathbf{D}^{\prime}$ is an $M \times M$ diagonal matrix. Let $\underline{r}=\left(r_{1}, r_{2}, \ldots, r_{M}\right)$ and $\underline{d}^{\prime}=\left(d_{1}^{\prime}, d_{2}^{\prime}, \ldots, d_{M}^{\prime}\right)$ denote the diagonal entries of $\mathbf{R}$ and $\mathbf{D}^{\prime}$, respectively. From (9), we know that $r_{k}^{2}=d_{k}^{\prime}$ for all $k=1,2, \ldots, M$. Furthermore, using the uniqueness property of the Cholesky factorization, it can be seen that the entries of the sequence $\underline{d}^{\prime}$ exactly represent the Cholesky values of $\mathbf{A}$.

Definition 1: Let $\underline{b}=\left(b_{1}, b_{2}, \ldots, b_{M}\right)$ and $\underline{c}=\left(c_{1}, c_{2}, \ldots\right.$, $\left.c_{M}\right)$ be two positive real-valued sequences satisfying $b_{1} \geq b_{2} \geq$ $\cdots \geq b_{M}$ and $c_{1} \geq c_{2} \geq \cdots \geq c_{M}$. We say that $\underline{b}$ majorizes $\underline{c}$ in the product sense [20] if

$$
\prod_{k=1}^{l} b_{k} \geq \prod_{k=1}^{l} c_{k}
$$

for all $l=1,2, \ldots, M$ and with equality when $l=M$.

Lemma 1: For a positive-definite matrix $\mathbf{A}=\mathbf{H}_{p}^{H} \mathbf{H}_{p}$, the sequence $\underline{\sigma}$ majorizes the sequence $\underline{d}$ in the product sense, i.e.,

$$
\prod_{k=1}^{l} \sigma_{k} \geq \prod_{k=1}^{l} d_{k}
$$

for all $l=1,2, \ldots, M$ and with equality when $l=M$.

This lemma and its proof can be found in [18] and [20].

Assume that the entries of both $\underline{r}$ and $\underline{d}^{\prime}$ are also in descending order. Then, by Lemma 1 , we can obtain

$$
\prod_{k=1}^{l} \sigma_{k} \geq \prod_{k=1}^{l} d_{k}=\prod_{k=1}^{l} d_{k}^{\prime}=\prod_{k=1}^{l} r_{k}^{2}
$$

for all $l=1,2, \ldots, M$ and with equality when $l=M$. For a positive-definite matrix $\mathbf{A}=\mathbf{H}_{p}^{H} \mathbf{H}_{p}$, it is known that $\sigma_{k}=\lambda_{k}^{2}$, where $\lambda_{k}$ is the $k$ th largest singular value of $\mathbf{H}_{p}$. We thus have

$$
\prod_{k=1}^{l} \lambda_{k}^{2}=\prod_{k=1}^{l} \sigma_{k} \geq \prod_{k=1}^{l} r_{k}^{2}
$$

for all $l=1,2, \ldots, M$ and with equality when $l=M$. From (13), we arrive at that $\lambda_{M}^{2} \leq r_{M}^{2}$. Note that $r_{M}=[\mathbf{R}]_{\min }$. We can have $\lambda_{M} \leq[\mathbf{R}]_{\text {min }}$ since both $\lambda_{M}$ and $[\mathbf{R}]_{\text {min }}$ are positive values, which completes the proof.

\section{BASIS TRANSFORMATION MEthod}

In the previous section, we see that the lower bound obtained with the QRD-based method is tighter than that with the SVDbased method. In this section, we propose a method for further tightening the lower bound of the free distance. To do that, we first observe how the channel matrix affects the tightness of the bound in (8). From [17], it can be seen that when the diagonal entries of $\mathbf{R}$ are all equal, the equality in (8) will hold. We then conjecture that the tightness of the lower bound is related to the spread of the diagonal entries in the R-factor, which is defined as the $[\mathbf{R}]$-value spread. The $[\mathbf{R}]$-value spread is the value of $[\mathbf{R}]_{\max }$ divided by that of $[\mathbf{R}]_{\min }$, where $[\mathbf{R}]_{\max }$ is the maximum diagonal entry in the matrix $\mathbf{R}$. If the $[\mathbf{R}]$ value spread is smaller, then the bound in (8) is tighter. Now, considering the signal part in (1), we have

$$
\begin{aligned}
\mathbf{y}_{\mathrm{s}} & =\mathbf{H}_{p} \mathbf{x} \\
& =x_{1} \mathbf{h}_{p, 1}+x_{2} \mathbf{h}_{p, 2}+\cdots+x_{M} \mathbf{h}_{p, M}
\end{aligned}
$$

where $\mathbf{h}_{p, k}$ is the $k$ th column of $\mathbf{H}_{p}$. Thus, $\mathbf{y}_{\mathrm{s}}$ can be seen as a vector expanded by a basis formed by the columns of $\mathbf{H}_{p}$, i.e., $\left[\mathbf{h}_{p, 1}, \mathbf{h}_{p, 2}, \ldots, \mathbf{h}_{p, M}\right]$, and the corresponding coordinate is $\left[x_{1}, x_{2}, \ldots, x_{M}\right]^{T}$. With an invertible matrix $\mathbf{Z}$, we can rewrite (14) as

$$
\begin{aligned}
\mathbf{y}_{\mathrm{s}} & =\mathbf{H}_{p} \mathbf{Z Z}^{-1} \mathbf{x} \\
& =\overline{\mathbf{H}}_{p} \overline{\mathbf{x}}
\end{aligned}
$$

where $\overline{\mathbf{H}}_{p}=\mathbf{H}_{p} \mathbf{Z}$, and $\overline{\mathbf{x}}=\mathbf{Z}^{-1} \mathbf{x}$. Thus, the basis is transformed to the columns of $\overline{\mathbf{H}}_{p}$, and the corresponding coordinate is $\overline{\mathbf{x}}=\left[\bar{x}_{1}, \bar{x}_{2}, \ldots, \bar{x}_{M}\right]^{T}$. If a proper $\mathbf{Z}$ can be chosen such that the $[\mathbf{R}]$-value spread of the R-factor in $\overline{\mathbf{H}}_{p}$ is reduced, then the bound in (8) can be tightened. This is the basic concept of our basis transformation method.

Using the idea previously described, we rewrite the free distance as

$$
\begin{aligned}
d_{\text {free }} & =\min _{\mathbf{x}, \mathbf{x}^{\prime} \in \mathcal{X}^{M}, \mathbf{x} \neq \mathbf{x}^{\prime}}\left\|\mathbf{H}_{p}\left(\mathbf{x}-\mathbf{x}^{\prime}\right)\right\|^{2} \\
& =\min _{\mathbf{x}, \mathbf{x}^{\prime} \in \mathcal{X}^{M}, \mathbf{x} \neq \mathbf{x}^{\prime}}\left\|\mathbf{H}_{p} \mathbf{Z} \mathbf{Z}^{-1}\left(\mathbf{x}-\mathbf{x}^{\prime}\right)\right\|^{2} \\
& =\min _{\overline{\mathbf{x}}, \overline{\mathbf{x}}^{\prime} \in \overline{\mathcal{X}}^{M}, \overline{\mathbf{x}} \neq \overline{\mathbf{x}}^{\prime}}\left\|\overline{\mathbf{H}}_{p}\left(\overline{\mathbf{x}}-\overline{\mathbf{x}}^{\prime}\right)\right\|^{2}
\end{aligned}
$$

where $\overline{\mathcal{X}}^{M}$ is the symbol vector constellation reshaped by $\mathbf{Z}^{-1}$. In Appendix $A$, we show that the lower bound yielded by the SVD in (7) is still valid. However, $d_{\min }\left(\overline{\mathcal{X}}_{k}\right)$ becomes difficult to find since in general $d_{\min }\left(\overline{\mathcal{X}}^{M}\right) \neq$ $\min \left\{d_{\min }\left(\overline{\mathcal{X}}_{1}\right), \ldots d_{\min }\left(\overline{\mathcal{X}}_{M}\right)\right\}$, where $d_{\min }\left(\overline{\mathcal{X}}_{k}\right)=\min \mid \bar{x}_{k}-$ $\left.\bar{x}_{k}^{\prime}\right|^{2}$. On the other hand, the lower bound yielded by the QRD in (8) is no longer valid. From [17], we see that the lower bound in (8) is derived with the assumption of (6). Thus, if let $d_{\min }\left(\overline{\mathcal{X}}^{1}\right)=\min \left\{d_{\min }\left(\overline{\mathcal{X}}_{1}\right), d_{\min }\left(\overline{\mathcal{X}}_{2}\right), \cdots d_{\min }\left(\overline{\mathcal{X}}_{M}\right)\right\}$, then we can have a new lower bound from (8) as

$$
d_{\text {free }} \geq[\overline{\mathbf{R}}]_{\min }^{2} d_{\min }\left(\overline{\mathcal{X}}^{1}\right) .
$$

As mentioned, $d_{\min }\left(\overline{\mathcal{X}}^{1}\right) \neq d_{\min }\left(\mathcal{X}^{M}\right)$ in general. We cannot conclude that (17) is tighter than (8) even when $[\overline{\mathbf{R}}]_{\min }^{2} \geq$ $[\mathbf{R}]_{\min }^{2}$. The other problem with (17) is that the value of $d_{\text {min }}\left(\overline{\mathcal{X}}^{1}\right)$ is no longer easy to obtain. This is because after the transformation, the signal constellation in each dimension 
is significantly expanded. The challenge in the basis transformation method is to find $\mathbf{Z}$ such that $d_{\min }\left(\overline{\mathcal{X}}^{1}\right)$ can easily be computed and at the same time $d_{\min }\left(\overline{\mathcal{X}}^{1}\right)=d_{\min }\left(\mathcal{X}^{M}\right)$. The constraints are stringent and cannot be satisfied by most of the transformations. Fortunately, we have found two matrices that can do the job.

\section{A. Permutation Matrix}

The first transformation matrix we propose is the permutation matrix. For a given $N_{r} \times M$ candidate channel matrix $\mathbf{H}_{p}$, we can have $M$ ! different column permutation patterns. Let $\Pi_{i}$ denote the permutation matrix corresponding to the $i$ th permutation pattern, where $1 \leq i \leq M$ !. We can rewrite the free distance as

$$
\begin{aligned}
d_{\text {free }} & =\min _{\mathbf{x}, \mathbf{x}^{\prime} \in \mathcal{X}^{M}, \mathbf{x} \neq \mathbf{x}^{\prime}}\left\|\mathbf{H}_{p} \boldsymbol{\Pi}_{i} \boldsymbol{\Pi}_{i}^{-1}\left(\mathbf{x}-\mathbf{x}^{\prime}\right)\right\|^{2} \\
& =\min _{\mathbf{x}, \mathbf{x}^{\prime} \in \mathcal{X}^{M}, \mathbf{x} \neq \mathbf{x}^{\prime}}\left\|\mathbf{H}_{p} \boldsymbol{\Pi}_{i} \boldsymbol{\Pi}_{i}^{T}\left(\mathbf{x}-\mathbf{x}^{\prime}\right)\right\|^{2}
\end{aligned}
$$

where $\Pi_{i}^{T}=\Pi_{i}^{-1}$ since $\Pi_{i}$ is an orthogonal matrix. Note that the combinations of $\boldsymbol{\Pi}_{i}^{T}\left(\mathbf{x}-\mathbf{x}^{\prime}\right)$ are the same as those of $(\mathbf{x}-$ $\mathbf{x}^{\prime}$ ), which allows us to rewrite (18) as

$$
d_{\text {free }}=\min _{\mathbf{x}, \mathbf{x}^{\prime} \in \mathcal{X}^{M}, \mathbf{x} \neq \mathbf{x}^{\prime}}\left\|\mathbf{H}_{p} \boldsymbol{\Pi}_{i}\left(\mathbf{x}-\mathbf{x}^{\prime}\right)\right\|^{2} .
$$

Let $\mathbf{R}_{i}$ denote the R-factor of $\mathbf{H}_{p} \boldsymbol{\Pi}_{i}$. The free distance can be bounded as

$$
d_{\text {free }} \geq\left[\mathbf{R}_{i}\right]_{\min }^{2} d_{\min }\left(\mathcal{X}^{M}\right) .
$$

For a given $\mathbf{H}_{p}$, we can obtain $M$ ! different R-factors with $M$ ! permutations. Denote $\mathbf{R}_{\text {per }}$ as the $\mathbf{R}$-factor having the largest minimum diagonal entry. Then, the minimum diagonal entry of $\mathbf{R}_{\text {per }}$ can be used in the lower bound in (8). Then, we have

$$
d_{\text {free }} \geq\left[\mathbf{R}_{\text {per }}\right]_{\min }^{2} d_{\min }\left(\mathcal{X}^{M}\right)
$$

where $\left[\mathbf{R}_{\text {per }}\right]_{\min }=\max \left(\left[\mathbf{R}_{1}\right]_{\min },\left[\mathbf{R}_{2}\right]_{\min }, \ldots,\left[\mathbf{R}_{M !}\right]_{\min }\right)$. In other words, we find the optimum permutation pattern such that the smallest $[\mathbf{R}]$-value spread can be obtained. We summarize the proposed basis transformation method with the permutation matrix (which is denoted as QRD-based BT-P) in Table I. Assume that $\boldsymbol{\Pi}_{1}=\mathbf{I}_{M}$. Then, $\left[\mathbf{R}_{1}\right]_{\min }=[\mathbf{R}]_{\min }$. Clearly, we can have the following inequality:

$$
\left[\mathbf{R}_{\mathrm{per}}\right]_{\min } \geq[\mathbf{R}]_{\min } .
$$

The inequality in (22) indicates that this permutation method can improve the tightness of (8).

\section{B. Transformation Matrix in $L R$}

The second transformation matrix we found is the basis transformation matrix used in LR. The LR technique has been successfully applied to MIMO systems for enhancing the detection performance [8], [9]. The basic idea is to find a new basis for the transmitted symbol vector, and then, signal detection is conducted in the basis-transformed domain. In
TABLE I

Algorithm of Proposed QRD-BASEd BT-P MeTHod

\begin{tabular}{l}
\hline \hline Algorithm 1: QRD-based BT-P Design \\
(1) For each candidate channel matrix $\mathbf{H}_{p}$, \\
compute $\mathbf{H}_{p, i}=\mathbf{H}_{p} \mathbf{\Pi}_{i}$ where $i=1,2, \ldots, M$ !; \\
(2) for $i=1: M !$ \\
(3) $\quad\left[\mathbf{Q}_{i}, \mathbf{R}_{i}\right]=\mathrm{QR}$ Decomposition $\left(\mathbf{H}_{p, i}\right)$; \\
(4) $\left[\mathbf{R}_{i}\right]_{\min }=\operatorname{minimum}$ diagonal entry of $\mathbf{R}_{i}$; \\
(5) end \\
(6) $\left[\mathbf{R}_{\text {per }}\right]_{\text {min }}=\max \left(\left[\mathbf{R}_{1}\right]_{\min },\left[\mathbf{R}_{2}\right]_{\min }, \cdots,\left[\mathbf{R}_{M !}\right]_{\min }\right)$; \\
(7) Let $\left[\mathbf{R}_{\text {per }}\right]_{\min }$ be the minimum diagonal entry of the $\mathrm{R}-$ factor for $\mathbf{H}_{p}$; \\
(8) Choose the antenna subset whose $\mathbf{H}_{p}$ has the largest $\left[\mathbf{R}_{\text {per }}\right]_{\text {min }}$;
\end{tabular}
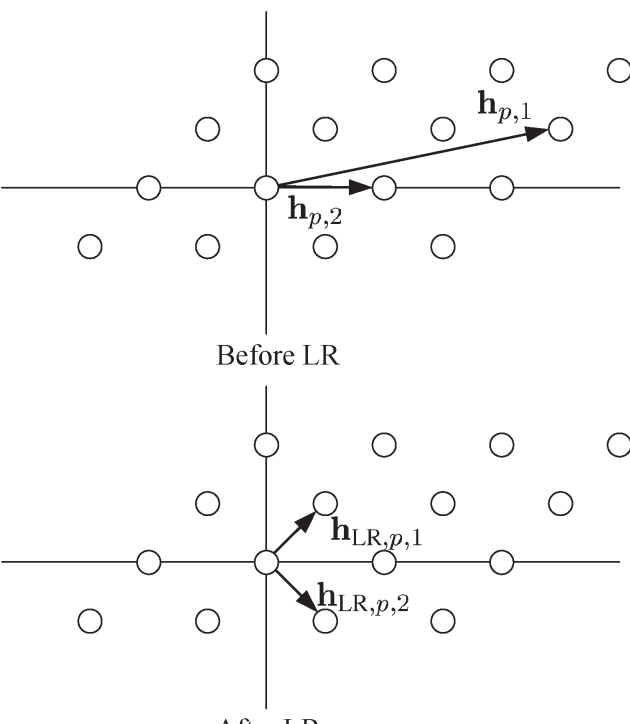

After LR

Fig. 2. $\quad$ LR for $M=2$.

this paper, we propose using the transformation matrix used in LR to obtain a tighter lower bound of the free distance. Note that the ML detection is still conducted in the original basis. With $\mathbf{H}_{p}=\left[\mathbf{h}_{p, 1}, \mathbf{h}_{p, 2}, \ldots, \mathbf{h}_{p, M}\right]$, we can describe an $M$-dimensional lattice $L$ as

$$
L\left\{\mathbf{h}_{p, 1}, \mathbf{h}_{p, 2}, \ldots, \mathbf{h}_{p, M}\right\}=\sum_{k=1}^{M} s_{k} \mathbf{h}_{p, k}
$$

where $s_{k}$ is a complex integer. The vector set $\left\{\mathbf{h}_{p, 1}, \mathbf{h}_{p, 2}, \ldots\right.$, $\left.\mathbf{h}_{p, M}\right\}$ is a basis spanning $L$. Let $\mathbf{P}$ be an invertible matrix whose entries are all complex integers, and we have

$$
\begin{aligned}
\mathbf{H}_{\mathrm{LR}, p} & =\mathbf{H}_{p} \mathbf{P} \\
& =\left[\mathbf{h}_{\mathrm{LR}, p, 1}, \mathbf{h}_{\mathrm{LR}, p, 2}, \ldots, \mathbf{h}_{\mathrm{LR}, p, M}\right] .
\end{aligned}
$$

Note that all the entries of $\mathbf{P}^{-1}$ are also complex integers. Thus, the column vectors of the matrix $\mathbf{H}_{\mathrm{LR}, p}$ also form a basis for the same lattice. The LR method finds a basis whose elements are as orthogonal as possible, and at the same time, the magnitudes of the basis elements are as short as possible. An example of LR with $M=2$ is illustrated in Fig. 2. As we can see, the reduced basis vectors will have a shorter length, and the orthogonality of $\mathbf{H}_{\mathrm{LR}, p}$ is improved as well. Several algorithms to implement LR have been proposed in the literature. Among them, the complex Lenstra-Lenstra-Lovász (CLLL) algorithm 
TABLE II

OPERATIONS OF CLLL ALGORITHM

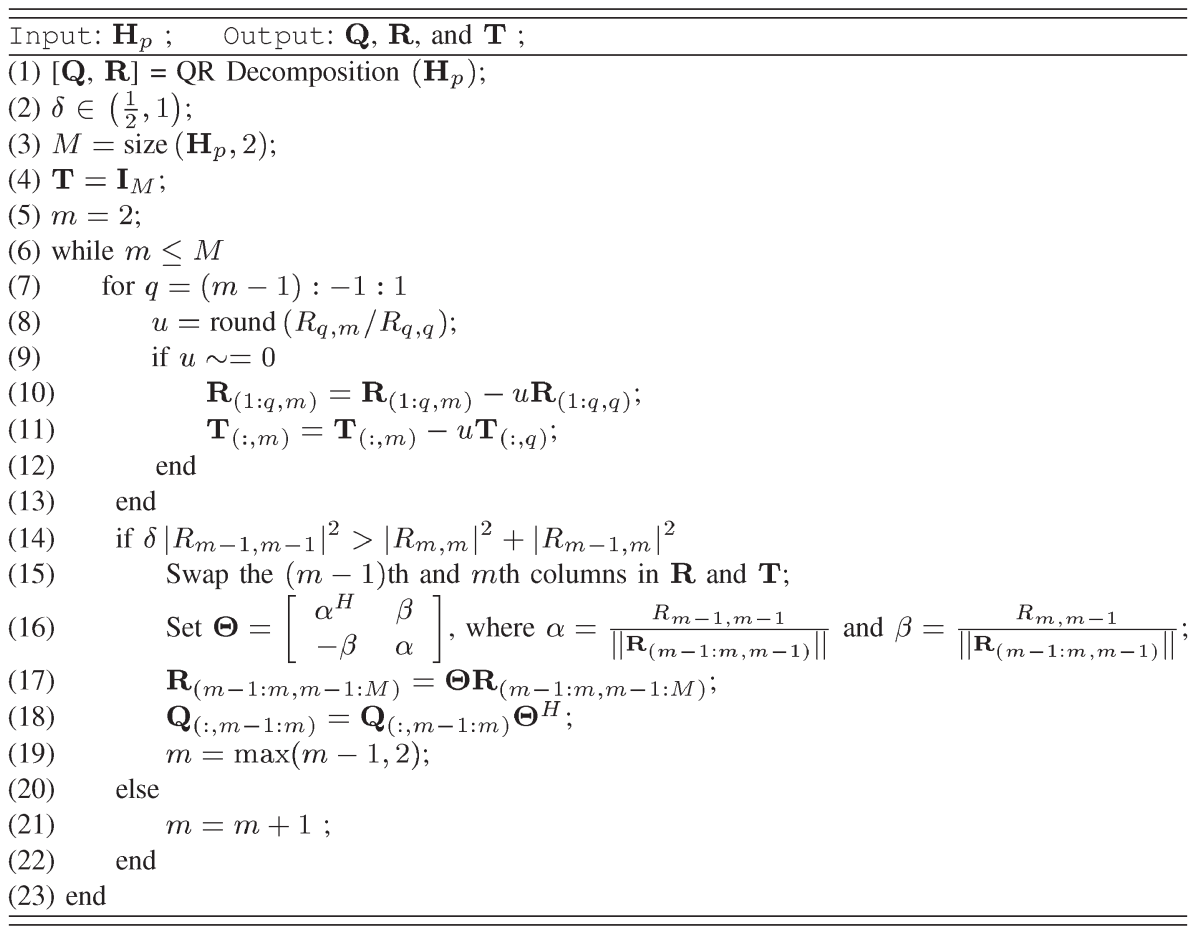

is most widely used since its computational complexity is lower. We then use the CLLL as our LR algorithm in this paper. The operation of the CLLL algorithm is summarized in Table II. As we can see in the table, a parameter $\delta \in(0.5,1)$ is defined in the CLLL. This parameter determines the orthogonality of the transformed channel matrix $\mathbf{H}_{\mathrm{LR}, p}$. A larger $\delta$ will make $\mathbf{H}_{\mathrm{LR}, p}$ closer to an orthogonal matrix. However, the CLLL algorithm will require more iterations to converge, and its computational complexity is higher.

Now, we can conduct $\mathrm{LR}$ on $\mathbf{H}_{p}$ to obtain $\mathbf{P}$. Rewrite the free distance in (3) as

$$
\begin{aligned}
d_{\text {free }} & =\min _{\mathbf{x}, \mathbf{x}^{\prime} \in \mathcal{X}^{M}, \mathbf{x} \neq \mathbf{x}^{\prime}}\left\|\mathbf{H}_{p} \mathbf{P} \mathbf{P}^{-1}\left(\mathbf{x}-\mathbf{x}^{\prime}\right)\right\|^{2} \\
& =\min _{\mathbf{x}_{\mathrm{LR}}, \mathbf{x}_{\mathrm{LR}}^{\prime} \in \mathcal{X}_{\mathrm{LR}}^{M}, \mathbf{x}_{\mathrm{LR}} \neq \mathbf{x}_{\mathrm{LR}}^{\prime}}\left\|\mathbf{H}_{\mathrm{LR}, p}\left(\mathbf{x}_{\mathrm{LR}}-\mathbf{x}_{\mathrm{LR}}^{\prime}\right)\right\|^{2}
\end{aligned}
$$

where $\mathbf{x}_{\mathrm{LR}}-\mathrm{x}_{\mathrm{LR}}^{\prime}=\mathbf{P}^{-1}\left(\mathbf{x}-\mathbf{x}^{\prime}\right)$, and $\mathcal{X}_{\mathrm{LR}}^{M}$ represents the symbol vector constellation after the transformation. Thus, we can conduct the $\mathrm{QRD}$ on $\mathbf{H}_{\mathrm{LR}, p}$, obtaining $\mathbf{H}_{\mathrm{LR}, p}=\mathbf{Q}_{\mathrm{LR}} \mathbf{R}_{\mathrm{LR}}$. Using (17), we can have a lower bound of the free distance as

$$
d_{\text {free }} \geq\left[\mathbf{R}_{\mathrm{LR}}\right]_{\min }^{2} d_{\min }\left(\mathcal{X}_{\mathrm{LR}}^{1}\right)
$$

where $d_{\min }\left(\mathcal{X}_{\mathrm{LR}}^{1}\right)$ is expressed as

$$
\begin{aligned}
& d_{\min }\left(\mathcal{X}_{\mathrm{LR}}^{1}\right) \\
& =\min \left\{d_{\min }\left(\mathcal{X}_{\mathrm{LR}, 1}\right), d_{\min }\left(\mathcal{X}_{\mathrm{LR}, 2}\right), \ldots, d_{\min }\left(\mathcal{X}_{\mathrm{LR}, M}\right)\right\} .
\end{aligned}
$$

In Appendix $\mathrm{B}$, we show that $d_{\min }\left(\mathcal{X}_{\mathrm{LR}}^{1}\right)$ can be seen as equal to $d_{\min }\left(\mathcal{X}^{M}\right)$. As a result, only $\left[\mathbf{R}_{\mathrm{LR}}\right]_{\min }$ needs to be evaluated in the comparison of (26) and (8).
Proposition 2: For an $N_{r} \times M$ full column-rank matrix $\mathbf{H}_{p}$, if LR is conducted with the CLLL algorithm, the following inequality is held:

$$
\left[\mathbf{R}_{\mathrm{LR}}\right]_{\min } \geq[\mathbf{R}]_{\min } .
$$

Proof: See Appendix C.

Thus, for a candidate channel matrix, the lower bound obtained with (26) will be always tighter than or equal to the original QRD-based lower bound. We can then select the antenna subset whose corresponding channel matrix has the maximum $\left[\mathbf{R}_{\mathrm{LR}}\right]_{\min }$. Note that the singular values of a matrix are invariant under the column permutation operation. Therefore, the tightness of the SVD-based lower bound cannot be improved by the transformation with permutation matrices. As for the transformation with the LR matrix $\mathbf{P}$, the minimum singular value may also be enlarged. However, as proved in Proposition 1, the resultant minimum singular value will still be smaller than $\left[\mathbf{R}_{\mathrm{LR}}\right]_{\min }$.

\section{Cascade of Permutation and LR Matrices}

To further improve performance, we can consider the cascade of a permutation and an LR matrix as another transformation matrix. Let $\mathbf{S}_{i}=\mathbf{P} \boldsymbol{\Pi}_{i}$. The free distance can then be expressed as

$$
\begin{aligned}
d_{\text {free }} & =\min _{\mathbf{x}, \mathbf{x}^{\prime} \in \mathcal{X}^{M}, \mathbf{x} \neq \mathbf{x}^{\prime}}\left\|\mathbf{H}_{p} \mathbf{S}_{i} \mathbf{S}_{i}^{-1}\left(\mathbf{x}-\mathbf{x}^{\prime}\right)\right\|^{2} \\
& =\min _{\mathbf{x}, \mathbf{x}^{\prime} \in \mathcal{X}^{M}, \mathbf{x} \neq \mathbf{x}^{\prime}}\left\|\mathbf{H}_{p} \mathbf{P} \boldsymbol{\Pi}_{i} \boldsymbol{\Pi}_{i}^{T} \mathbf{P}^{-1}\left(\mathbf{x}-\mathbf{x}^{\prime}\right)\right\|^{2} .
\end{aligned}
$$

With the effective channel matrix $\mathbf{H}_{p} \mathbf{P} \mathbf{\Pi}_{i}$, the QRD-based lower bound of the free distance can be tightened even further. 
TABLE III

Algorithm of Proposed QRD-BAsed BT-C METhod

Algorithm 2: QRD-based BT-C Design

(1) For each candidate channel matrix $\mathbf{H}_{p}, \mathbf{P}=\operatorname{CLLL}\left(\mathbf{H}_{p}\right)$;

(2) for $i=1: M$ !

(3) $\left[\mathbf{Q}_{\mathrm{LR}, i}, \mathbf{R}_{\mathrm{LR}, i}\right]=$ QR Decomposition $\left(\mathbf{H}_{p} \mathbf{P \Pi}_{i}\right)$;

(4) $\left[\mathbf{R}_{\mathrm{LR}, i}\right]_{\min }=$ minimum diagonal entry of $\mathbf{R}_{\mathrm{LR}, i}$;

(5) end

(6) $\left[\mathbf{R}_{\mathrm{cas}}\right]_{\min }=\max \left(\left[\mathbf{R}_{\mathrm{LR}, 1}\right]_{\min },\left[\mathbf{R}_{\mathrm{LR}, 2}\right]_{\min }, \cdots,\left[\mathbf{R}_{\mathrm{LR}, M !}\right]_{\min }\right)$;

(7) Let $\left[\mathbf{R}_{\mathrm{cas}}\right]_{\min }$ be the minimum diagonal entry of the R-factor for $\mathbf{H}_{p}$; (8) Choose the antenna subset whose $\mathbf{H}_{p}$ has the largest $\left[\mathbf{R}_{\mathrm{cas}}\right]_{\text {min }}$;

In this paper, we denote QRD-based BT-C as the proposed QRD-based method with the basis transformation using the cascade of permutation and LR matrices. Table III summarizes the operations of the proposed QRD-based BT-C method. Denote $\mathbf{R}_{\mathrm{LR}, i}$ as the R-factor of $\mathbf{H}_{p} \mathbf{P} \boldsymbol{\Pi}_{i}$ and $\left[\mathbf{R}_{\text {cas }}\right]_{\text {min }}$ as the $\mathbf{R}$-factor having the largest minimum diagonal entry among all $\mathbf{R}_{\mathrm{LR}, i}$ 's. In other words, $\left[\mathbf{R}_{\mathrm{cas}}\right]_{\min }=\max \left(\left[\mathbf{R}_{\mathrm{LR}, 1}\right]_{\min }\right.$, $\left.\left[\mathbf{R}_{\mathrm{LR}, 2}\right]_{\min }, \ldots,\left[\mathbf{R}_{\mathrm{LR}, M !}\right]_{\min }\right)$. Thus, we can have the following inequality:

$$
\left[\mathbf{R}_{\mathrm{cas}}\right]_{\min } \geq\left[\mathbf{R}_{\mathrm{LR}}\right]_{\min }
$$

From (31) and (22), we see that the lower bound of $\left[\mathbf{R}_{\text {cas }}\right]_{\min }$ is larger than that of $\left[\mathbf{R}_{\text {per }}\right]_{\text {min }}$ since $\left[\mathbf{R}_{\mathrm{LR}}\right]_{\min } \geq[\mathbf{R}]_{\text {min }}$. This, however, does not imply that $\left[\mathbf{R}_{\text {cas }}\right]_{\text {min }} \geq\left[\mathbf{R}_{\text {per }}\right]_{\text {min }}$. We can only say that the probability of $\left[\mathbf{R}_{\text {cas }}\right]_{\text {min }} \geq\left[\mathbf{R}_{\text {per }}\right]_{\text {min }}$ is larger. Furthermore, we can exchange the cascading order. In other words, we can let the transformation matrix be $\mathbf{S}_{i}=\boldsymbol{\Pi}_{i} \mathbf{P}_{i}$. In this case, however, we have to conduct $\mathrm{LR}$ for each $\mathbf{H}_{p} \boldsymbol{\Pi}_{i}$, and the resultant computational complexity is then higher. Finally, we can even use a transformation matrix by cascading a series of permutation and LR matrices. For example, we can have a transformation matrix in the form of $\mathbf{P}_{1} \boldsymbol{\Pi}_{i} \mathbf{P}_{2} \boldsymbol{\Pi}_{i^{\prime}}$, where $1 \leq$ $i \leq M$ !, and $1 \leq i^{\prime} \leq M$ !. Simulations show that this may not be required. With only one-level cascading, the performance of the selection is very close to the optimum solution.

\section{IMPLEMENTATION ISSUES AND COMPLEXITY COMPARISON}

The computational complexity of the basis transformation method will be increased due to extra transformations and QRD operations. To reduce the computational complexity, we proposed several efficient methods for real-world implementations.

\section{A. Givens Rotation Method}

To reduce the computational complexity of the QRDs, we propose using Givens rotations [21] to compute each $\left[\mathbf{R}_{i}\right]_{\min }$. Assume that $\mathbf{H}_{p, 1}=\mathbf{Q}_{1} \mathbf{R}_{1}$ is available via a complete $\mathrm{QRD}$. Let $\mathbf{H}_{p, 2}$ be another matrix obtained by exchanging two neighbor columns of $\mathbf{H}_{p, 1}$. We seek to find $\mathbf{R}_{2}$ of $\mathbf{H}_{p, 2}$ without using another complete QRD. Denote $\check{\Pi}$ as a permutation matrix conducting a column-exchange operation on two neighbor columns, i.e.,

$$
\mathbf{H}_{p, 2}=\mathbf{Q}_{1} \mathbf{R}_{1} \check{\mathbf{\Pi}}=\mathbf{Q}_{1} \check{\mathbf{R}}_{1}
$$

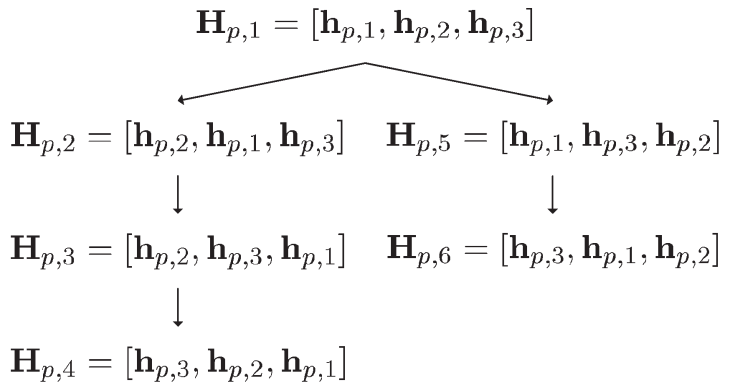

Fig. 3. $\left[\mathbf{R}_{i}\right]_{\min }$ computations for $M=3$.

where $\check{\mathbf{R}}_{1}$ is a near upper triangular matrix. Now, all we have to do is to transform $\check{\mathbf{R}}_{1}$ into an upper triangular matrix again. Since $\check{\Pi}$ only exchanges two neighbor columns of $\mathbf{R}_{1}$, we can upper triangulize $\check{\mathbf{R}}_{1}$ by a simple Givens rotation matrix $\mathbf{G}_{1}$. Then, $\mathbf{G}_{1} \check{\mathbf{R}}_{1}=\mathbf{T}$, where $\mathbf{T}$ is an upper triangular matrix. Thus, we can rewrite (32) as

$$
\mathbf{H}_{p, 2}=\mathbf{Q}_{1} \mathbf{G}_{1}^{H} \mathbf{G}_{1} \check{\mathbf{R}}_{1}=\mathbf{Q}_{2} \mathbf{T}
$$

where $\mathbf{Q}_{2}=\mathbf{Q}_{1} \mathbf{G}_{1}^{H}$ is a unitary matrix. From (33), we know that $\mathbf{Q}_{2} \mathbf{T}$ is the $\mathrm{QRD}$ of $\mathbf{H}_{p, 2}$, and $\mathbf{T}$ is equal to $\mathbf{R}_{2}$. In other words, we obtain $\mathbf{R}_{2}$ by simply left multiplying a Givens rotation matrix on $\check{\mathbf{R}}_{1}$ rather than by performing a complete QRD on $\mathbf{H}_{p, 2}$. Therefore, we can dramatically reduce the computational complexity of the proposed basis transformation method when conducting the permutation operations. Note that applying Givens rotations will not affect the performance of the proposed selection methods. Fig. 3 illustrates an example (for $M=3$ ) of how each $\left[\mathbf{R}_{i}\right]_{\min }$ can be derived with Givens rotations.

\section{B. Efficient Permutations}

Consider a Rayleigh flat-fading MIMO channel matrix $\mathbf{H}_{p}$. With the QRD, we have $\mathbf{H}_{p}=\mathbf{Q R}$. It has been shown that the square value of each diagonal entry in $\mathbf{R}$ is independently distributed with Gamma distribution [22]. That is, $[\mathbf{R}]_{k}^{2} \sim G(M+1-k)$, where $G(M+1-k)$ denotes the Gamma distribution with mean $\mathbb{E}\left[[\mathbf{R}]_{k}^{2}\right]=(M+1-k)$. Thus, the expectation of $[\mathbf{R}]_{M}^{2}$, which is equal to 1 , is the smallest. As a result, $[\mathbf{R}]_{M}$ has the highest probability to be the minimum diagonal entry of $\mathbf{R}$. In addition, note that Givens rotations have to be conducted sequentially. Using these two properties, we propose an efficient permutation method to reduce the required computational complexity. The idea is to conduct permutations in a local rather than global manner. Define an integer $\bar{M}$ and $\bar{M}<M$. With Givens rotations, permutating the first $\bar{M}$ columns of $\mathbf{H}_{p}$ will not change the resultant values of $\left([\mathbf{R}]_{\bar{M}+1},[\mathbf{R}]_{\bar{M}+2}, \ldots,[\mathbf{R}]_{M}\right)$. This is to say, if $[\mathbf{R}]_{M}$ is the minimum value, then these permutations are totally useless. Thus, we choose the last $M-\bar{M}$ columns of $\mathbf{H}_{p}$ for local permutations. If $\bar{M}$ is chosen to be much smaller than $M$, then the computational complexity can be reduced significantly. Note that local permutations will result in some performance loss. In [22] and [23], it has theoretically been shown that $\mathbb{E}\left[[\mathbf{R}]_{M}^{2}\right]$ tends to be larger when the columns of $\mathbf{H}_{p}$ are exchanged 
TABLE IV

Algorithm of Proposed QRD-Based BT-E Method

Algorithm 3: QRD-based BT-E Design

(1) For each candidate channel matrix $\mathbf{H}_{p}, \mathbf{P}=\operatorname{CLLL}\left(\mathbf{H}_{p}\right)$;

(2) Choose $\bar{M}<M$;

(3) for $i=1: \bar{M}$ !

(4) $\left[\mathbf{Q}_{\mathrm{LR}, i}, \mathbf{R}_{\mathrm{LR}, i}\right]=\mathrm{QR}$ Decomposition $\left(\mathbf{H}_{p} \mathbf{P} \Pi_{i}\right)$;

(5) $\left[\mathbf{R}_{\mathrm{LR}, i}\right]_{\text {min }}=$ minimum diagonal entry of $\mathbf{R}_{\mathrm{LR}, i}$;

(6) end

(7) $\left[\mathbf{R}_{\text {cas }}^{\text {eff }}\right]_{\min }=\max \left(\left[\mathbf{R}_{\mathrm{LR}, 1}\right]_{\min },\left[\mathbf{R}_{\mathrm{LR}, 2}\right]_{\min }, \cdots,\left[\mathbf{R}_{\mathrm{LR}, \bar{M} !}\right]_{\min }\right)$;

(8) Let $\left[\mathbf{R}_{\text {cas }}^{\text {eff }}\right]_{\text {min }}$ be the minimum diagonal entry of the R-factor for $\mathbf{H}_{p}$; (9) Choose the antenna subset whose $\mathbf{H}_{p}$ has the largest $\left[\mathbf{R}_{\mathrm{cas}}^{\mathrm{eff}}\right]_{\mathrm{min}}$;

according to the norm-ascending order. This is referred to as preordering. Thus, we can combine this preordering with our efficient permutation method to compensate the performance loss.

The idea of efficient permutation can also be applied to the QRD-based BT-C scheme. As shown in Appendix C, the $[\mathbf{R}]$-value spread of $\mathbf{R}_{\mathrm{LR}}$ will effectively be reduced with the CLLL algorithm. Thus, the values of the diagonal entries in $\mathbf{R}_{\mathrm{LR}}$ can become very close. Preordering is then not required. From the simulations, we found that due to some special properties of the CLLL, using the first $\bar{M}$ neighbor columns for local permutations can provide slightly better performance. For this reason, when conducting efficient permutations for the QRD-based BT-C scheme, we choose the first $\bar{M}$ columns of $\mathbf{H}_{p} \mathbf{P}$. The operation of the QRD-based BT-C method implemented with efficient permutations (which is denoted as QRD-based BT-E) is shown in Table IV.

\section{Complexity Comparison}

In this paper, we use the number of floating operations (FLOPS) required in an algorithm as the measure for the computational complexity. Many algorithms for conducting the QRD and SVD have been developed [21]. In general, the QRD requires fewer FLOPS than the SVD does [21], [24]. Thus, the QRD-based selection scheme not only has better performance but also requires lower computational complexity. In Table V, we summarize the order of the computational complexity in each proposed method.

As we can see, computing $\left[\mathbf{R}_{i}\right]_{\min }$ for $i=2, \ldots, M$ ! in the QRD-based BT-P method requires $\mathcal{O}\left(M^{3} M\right.$ !) FLOPS, which can be reduced to $\mathcal{O}(M M !)$ with Givens rotations. It has been shown that the computational complexity of the CLLL algorithm is $\mathcal{O}\left(M^{4}\right)$ [25]. Therefore, the total computational complexity of the QRD-based BT-C scheme with Given rotations is $\mathcal{O}\left(M^{4}+M M\right.$ !). The term $M M$ ! may grow rapidly when $M$ becomes very large, which dominates the total computational complexity. This problem can be solved with the efficient permutation method proposed in Section IV-B. In the QRD-based BT-E scheme, $\bar{M}$ can be chosen much smaller than $M$, and the resultant computational complexity can further be reduced to $\mathcal{O}\left(M^{4}+M \bar{M} !\right) \cong \mathcal{O}\left(M^{4}\right)$. Although the required complexity will be increased when the LR is considered, the overall complexity is still much lower than that of the exhaustive search.

\section{OTHER APPLICATIONS}

\section{A. Receive and Joint Transmit/Receive Antenna Selection}

Antenna selection can also be conducted at the receiver. As we did in transmit antenna selection, we can select a subset of receive antennas according to a performance criterion. Here, we select $M$ out of $N_{r}$ receive antenna elements. Note that the receiver does not have to feed the index of the selected antenna subset back to the transmitter, which is a significant advantage. However, the performance of receive antenna selection may be inferior to transmit antenna selection for the same number of candidate channel matrices. We now give a simple example to illustrate this property. Consider a MIMO system with $N_{t}=2, N_{r}=3$, and $M=2$, where the channel matrix can be expressed as

$$
\mathbf{H}=\left[\begin{array}{ll}
h_{1,1} & h_{1,2} \\
h_{2,1} & h_{2,2} \\
h_{3,1} & h_{3,2}
\end{array}\right] .
$$

From the diversity point of view, we can treat a $3 \times 2$ MIMO channel $\mathbf{H}$ as two separate $3 \times 1$ subchannels, each of which is obtained from a column of $\mathbf{H}$. The reason is that either $x_{1}$ or $x_{2}$ has the same diversity as the original $3 \times 2$ MIMO system since the ML detection is adopted at the receiver. This facilitates a simple performance analysis for the antenna selection problem. For receive antenna selection, $x_{1}$ has three candidate channel columns for selection, which are denoted as

$$
\mathbf{h}_{1}=\left[\begin{array}{l}
h_{1,1} \\
h_{2,1}
\end{array}\right], \mathbf{h}_{2}=\left[\begin{array}{l}
h_{1,1} \\
h_{3,1}
\end{array}\right], \text { and } \mathbf{h}_{3}=\left[\begin{array}{l}
h_{2,1} \\
h_{3,1}
\end{array}\right] .
$$

It can be seen that there exists a correlation between any two columns since those candidate channel columns have the common entries. This correlation will degrade the performance of receive antenna selection.

Now, consider transmit antenna selection in a MIMO system with $N_{t}=3, N_{r}=2$ and $M=2$, where the channel matrix can be expressed as

$$
\mathbf{H}=\left[\begin{array}{lll}
h_{1,1} & h_{1,2} & h_{1,3} \\
h_{2,1} & h_{2,2} & h_{2,3}
\end{array}\right] .
$$

For transmit antenna selection, $x_{1}$ also has three candidate channel columns given by

$$
\mathbf{h}_{1}=\left[\begin{array}{l}
h_{1,1} \\
h_{2,1}
\end{array}\right], \mathbf{h}_{2}=\left[\begin{array}{l}
h_{1,2} \\
h_{2,2}
\end{array}\right], \text { and } \mathbf{h}_{3}=\left[\begin{array}{l}
h_{1,3} \\
h_{2,3}
\end{array}\right] .
$$

Note here that three independent columns are available for selection, which is different from receive antenna selection. This clearly indicates that transmit antenna selection can outperform receive antenna selection for the same number of candidate channel matrices. However, as mentioned, conducting antenna selection at the transmitter side requires feedback overhead. Thus, there is a tradeoff between the feedback requirement and diversity performance. In addition, note that increasing the number of receive antennas may not be always possible due to the receiver size constraint. Therefore, we can then consider joint transmit/receive antenna selection, 
TABLE V

COMPLEXITY COMPARISONS

\begin{tabular}{|l|c|c|c|c|}
\hline \hline & $\mathbf{P}$ & {$\left[\mathbf{R}_{1}\right]_{\min }$} & {$\left[\mathbf{R}_{i}\right]_{\min }$ for $i=2, \cdots, M !$} & Total \\
\hline QRD-based & $\backslash$ & $\mathcal{O}\left(M^{3}\right)$ & $\mathcal{O}$ & $\mathcal{O}\left(M^{3}\right)$ \\
\hline QRD-based BT-P & $\backslash$ & $\mathcal{O}\left(M^{3}\right)$ & $\mathcal{O}\left(M^{3} M !\right)$ & $\mathcal{O}\left(M^{3} M !\right)$ \\
\hline $\begin{array}{l}\text { QRD-based BT-P with } \\
\text { Givens rotations }\end{array}$ & $\backslash$ & $\mathcal{O}\left(M^{3}\right)$ & $\mathcal{O}(M M !)$ & $\mathcal{O}\left(M^{3}+M M !\right)$ \\
\hline \hline & $\mathbf{P}$ & {$\left[\mathbf{R}_{\mathrm{LR}, 1}\right]_{\min }$} & {$\left[\mathbf{R}_{\mathrm{LR}, i}\right]_{\min }$ for $i=2, \cdots, M !$} & Total \\
\hline QRD-based BT-C & $\mathcal{O}\left(M^{4}\right)$ & $\mathcal{O}\left(M^{3}\right)$ & $\mathcal{O}\left(M^{3} M !\right)$ & $\mathcal{O}\left(M^{4}+M^{3} M !\right)$ \\
\hline $\begin{array}{l}\text { QRD-based BT-C with } \\
\text { Givens rotations }\end{array}$ & $\mathcal{O}\left(M^{4}\right)$ & $\mathcal{O}\left(M^{3}\right)$ & $\mathcal{O}(M M !)$ & $\mathcal{O}\left(M^{4}+M M !\right)$ \\
\hline \hline & $\mathbf{P}$ & {$\left[\mathbf{R}_{\mathrm{LR}, 1}\right]_{\min }$} & {$\left[\mathbf{R}_{\mathrm{LR}, i}\right]_{\min }$ for $i=2, \cdots, \bar{M} !$} & $\mathcal{O}\left(M^{3} \bar{M} !\right)$ \\
\hline QRD-based BT-E & $\mathcal{O}\left(M^{4}\right)$ & $\mathcal{O}\left(M^{3}\right)$ & $\mathcal{O}(M \bar{M} !)$ & $\mathcal{O}\left(M^{4}+M \bar{M} !\right)$ \\
\hline $\begin{array}{l}\text { QRD-based BT-E with } \\
\text { Givens rotations }\end{array}$ & $\mathcal{O}\left(M^{4}\right)$ & $\mathcal{O}\left(M^{3}\right)$ & $\mathcal{O}\left(M^{4}\right)$ \\
\hline \hline
\end{tabular}

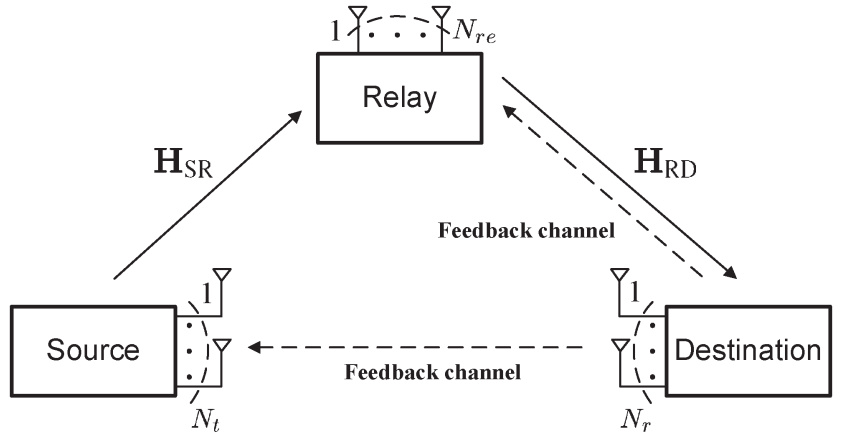

Fig. 4. System model of a two-hop AF MIMO relay system.

conducting antenna selection at both the transmitter and the receiver side simultaneously, to achieve the optimum tradeoff.

Consider an $N_{r} \times N_{t}$ MIMO channel $\mathbf{H}$, where $N_{r}>M$, and $N_{t}>M$. We have $\left(\begin{array}{c}N_{t} \\ M\end{array}\right) \times\left(\begin{array}{c}N_{r} \\ M\end{array}\right)$ possible candidate channel matrices. It is worth noting that we only need $\left\lceil\log _{2}\left(\begin{array}{c}N_{t} \\ M\end{array}\right)\right\rceil$ bits for feedback, where $\lceil t\rceil$ denotes the smallest integer larger than $t$. In addition, the joint selection scheme may provide more candidate channel matrices for a fixed number of total antennas. For example, if $N_{t}=4, N_{r}=4$, and $M=3$, then we have 16 candidate channel matrices, and only two bits are required for feedback. If we conduct pure transmit antenna selection with $N_{t}=5, N_{r}=3$, and $M=3$, then the number of the candidates is reduced to ten. Furthermore, the required bits for feedback will be increased to four. Note that the total numbers of antenna elements are the same for these two cases, i.e., $N_{t}+N_{r}=8$.

\section{B. Antenna Selection in MIMO Relay Channels}

Recently, cooperative communications have drawn a great deal of attention in wireless transmission. With the additional relay nodes, spatial diversity can effectively be enhanced. Multiple antennas can be placed at the source, the relays, and the destination. Such a cooperative system is referred to as a MIMO relay system. We now extend the proposed methods to antenna selection in MIMO relay systems. In this paper, we only consider a simple two-hop amplify-and-forward (AF) system, as illustrated in Fig. 4.

As mentioned, we can consider antenna selection at each node for performance improvement. Assume that the relay node is equipped with $N_{\text {re }}$ antennas. Let $\mathbf{H}_{\mathrm{SR}}$ be the $N_{\text {re }} \times N_{t}$ source-to-relay channel matrix and $\mathbf{H}_{\mathrm{RD}}$ be the $N_{r} \times N_{\text {re }}$ relay-to-destination channel matrix. In the AF relay scheme, signal transmission is divided into two phases. Denote $p_{1}$ and $p_{2}$ as the indices of the candidate channel matrices in Phases I and II, respectively. With antenna selection at all nodes, the source transmits the signal $\mathrm{x}$ to the relay through an $M \times M$ channel $\mathbf{H}_{\mathrm{SR}, p_{1}}$ during Phase I. Note that in a two-hop system, the destination cannot receive the transmitted signal from the source in Phase I. Thus, the received signal at the relay can be expressed as $\mathbf{y}_{1}=\mathbf{H}_{\mathrm{SR}, p_{1}} \mathbf{x}+\mathbf{n}_{\mathrm{SR}}$, where $\mathbf{n}_{\mathrm{SR}}$ is a white Gaussian noise vector. In Phase II, the relay amplifies and retransmits $\mathbf{y}_{1}$ through an $M \times M$ channel $\mathbf{H}_{\mathrm{RD}, p_{2}}$. The corresponding received signal at the destination, which is denoted by $\mathbf{y}_{2}$, can then be expressed as

$$
\mathbf{y}_{2}=\mathbf{H}_{\mathrm{RD}, p_{2}}\left(\mathbf{H}_{\mathrm{SR}, p_{1}} \mathbf{x}+\mathbf{n}_{\mathrm{SR}}\right)+\mathbf{n}_{\mathrm{RD}}
$$

where $\mathbf{n}_{\mathrm{RD}}$ is also a white Gaussian noise vector. Equivalently, (34) can be written as

$$
\mathbf{y}_{2}=\mathbf{H}_{\mathrm{eq}, p} \mathbf{x}+\mathbf{n}_{\mathrm{eq}}
$$

where $\mathbf{H}_{\mathrm{eq}, p}=\mathbf{H}_{\mathrm{RD}, p_{2}} \mathbf{H}_{\mathrm{SR}, p_{1}}, \quad \mathbf{n}_{\mathrm{eq}}=\mathbf{H}_{\mathrm{RD}, p_{2}} \mathbf{n}_{\mathrm{SR}}+\mathbf{n}_{\mathrm{RD}}$, and $p$ depends on $p_{1}$ and $p_{2}$. Note that the equivalent noise vector $\mathbf{n}_{\mathrm{eq}}$ is colored with the covariance matrix of

$$
\mathbf{K}_{p}=\sigma_{\mathrm{SR}}^{2}\left(\mathbf{H}_{\mathrm{RD}, p_{2}} \mathbf{H}_{\mathrm{RD}, p_{2}}^{H}+\frac{\sigma_{\mathrm{RD}}^{2}}{\sigma_{\mathrm{SR}}^{2}} \mathbf{I}_{M}\right)
$$

where $\sigma_{\mathrm{SR}}^{2}$ and $\sigma_{\mathrm{RD}}^{2}$ are the variances of $\mathbf{n}_{\mathrm{SR}}$ and $\mathbf{n}_{\mathrm{RD}}$, respectively. To conduct ML detection, the equivalent noise vector must be whitened, and this can be achieved by left multiplying a matrix $\mathbf{W}_{p}$ on $\mathbf{y}_{2}$, where

$$
\mathbf{W}_{p}=\left(\mathbf{H}_{\mathrm{RD}, p_{2}} \mathbf{H}_{\mathrm{RD}, p_{2}}^{H}+\frac{\sigma_{\mathrm{RD}}^{2}}{\sigma_{\mathrm{SR}}^{2}} \mathbf{I}_{M}\right)^{-\frac{1}{2}} .
$$

After the whitening process, the resultant received signal can be expressed as

$$
\mathbf{y}_{2}^{\prime}=\mathbf{W}_{p} \mathbf{H}_{\mathrm{eq}, p} \mathbf{x}+\mathbf{W}_{p} \mathbf{n}_{\mathrm{eq}}
$$

where $\mathbf{y}_{2}^{\prime}=\mathbf{W}_{p} \mathbf{y}_{2}$. Since the covariance of $\mathbf{W}_{p} \mathbf{n}_{\mathrm{eq}}$ becomes a scaled identity matrix $\sigma_{\mathrm{SR}}^{2} \mathbf{I}_{M}$, the selection schemes described 
in Sections II and III can directly be used to enhance the system performance.

\section{Sphere Decoding Algorithm (SDA)}

The SDA is an efficient method to realize the ML detection in MIMO systems. In this section, we demonstrate the use of our proposed selection methods for the SDA. Considering the signal model in (1), the idea of the SDA is to search a subset of $\mathcal{X}^{M}$ such that

$$
\left\|\mathbf{y}-\mathbf{H}_{p} \mathbf{x}\right\|^{2} \leq \gamma^{2}
$$

where $\gamma$ is the radius of the searching sphere. First, we conduct the QRD on $\mathbf{H}_{p}$, yielding $\mathbf{H}_{p}=\mathbf{Q R}$. Since $\mathbf{Q}$ is a unitary matrix, (39) can be rewritten as

$$
\begin{aligned}
\left\|\mathbf{y}-\mathbf{H}_{p} \mathbf{x}\right\|^{2} & =\|\mathbf{y}-\mathbf{Q R} \mathbf{x}\|^{2} \\
& =\left\|\mathbf{y}^{\prime}-\mathbf{R} \mathbf{x}\right\|^{2} \\
& \leq \gamma^{2}
\end{aligned}
$$

where $\mathbf{y}^{\prime}=\mathbf{Q}^{H} \mathbf{y}$. Let $y_{i}^{\prime}$ denote the $i$ th entry of $\mathbf{y}^{\prime}$. With the upper triangular structure of $\mathbf{R}$, we can further rewrite (40) as

$$
\begin{aligned}
\left\|\mathbf{y}-\mathbf{H}_{p} \mathbf{x}\right\|^{2}= & \left(y_{M}^{\prime}-R_{M, M} x_{M}\right)^{2} \\
& +\left(y_{M-1}^{\prime}-R_{M-1, M} x_{M}-R_{M-1, M-1} x_{M-1}\right)^{2} \\
& +\cdots \\
\leq & \gamma^{2}
\end{aligned}
$$

The expression of (41) allows a tree search operation, starting with $x_{M}$, for finding the solution candidates. Then, the candidate with the minimum distance is chosen as the output.

As we can see, the QRD operation is required in the SDA. Thus, the QRD processing unit can be shared with proposed antenna selection methods. However, if we adopt other selection methods such as the SVD- or capacity-based method [7], then extra circuits are required to conduct the SVD or calculate the channel capacity. Thus, with the proposed methods, the implementation complexity of the receiver can effectively be reduced. The capacity-based method, maximizing the capacity of the channel matrix, is described as follows. For a given candidate channel matrix $\mathbf{H}_{p}$, the channel capacity is expressed as

$$
C=\log _{2} \operatorname{det}\left(\mathbf{I}_{M}+\frac{\rho}{M} \mathbf{H}_{p}^{H} \mathbf{H}_{p}\right)
$$

where $\rho$ is the average SNR. The method then evaluates (42) for each candidate channel matrix and selects the antenna subset having the maximum channel capacity. The computational complexity of the capacity-based method is $\mathcal{O}\left(M^{3}\right)$, mainly arising from the matrix multiplication and the determinant computation in (42). One additional overhead for the method is that the estimation of the noise variance is required.

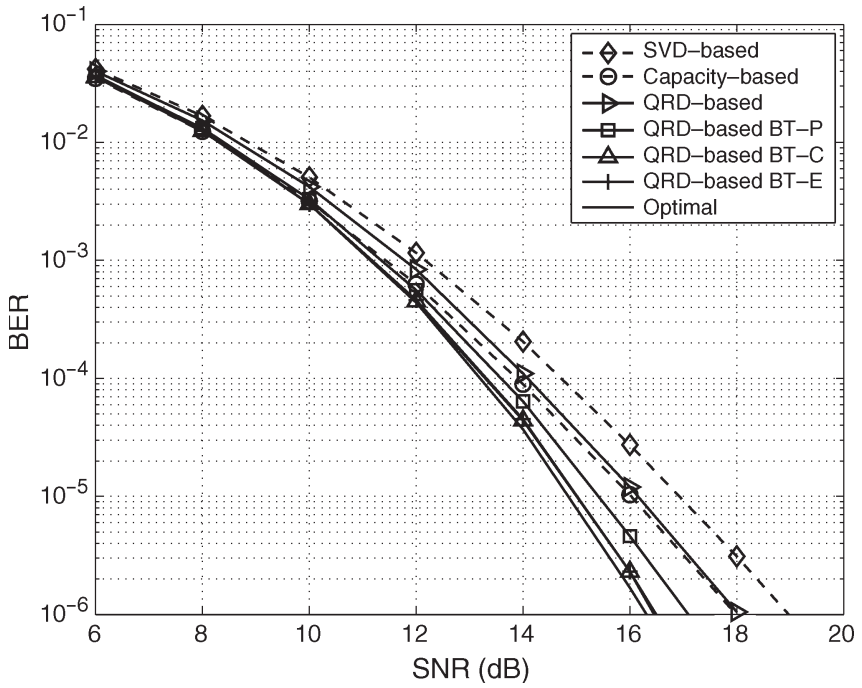

Fig. 5. BER performance comparison for transmit antenna selection $\left(N_{t}=6\right.$, $N_{r}=3$, and $M=3$ ).

\section{Simulation Results}

In this section, we report simulations evaluating the performance of our proposed selection methods. The simulation setup is described as follows. A flat-fading MIMO channel $\mathbf{H}$ is used; its entries are assumed to be i.i.d. complex Gaussian random variables with zero mean and unit variance. The modulation scheme is quadrature phase-shift keying (QPSK), and the detection method is ML. In addition, the parameter $\delta$ in the CLLL algorithm is set as 0.99 . In our simulations, several selection methods are compared, including 1) the SVD-based method, 2) the capacity-based method, 3) the QRD-based method, 4) the QRD-based BT-P method, 5) the QRD-based BT-C method, 6) the QRD-based BT-E method, and 7) the optimum method realized with an exhaustive search.

Fig. 5 shows the bit error rate (BER) performance of transmit antenna selection in the MIMO system. Here, $N_{t}=6$, $N_{r}=3$, and $M=3$. As we can see, the QRD-based method indeed outperforms the SVD-based method. The performance of the capacity-based method is comparable with that of the QRD-based scheme. However, the capacity-based method requires additional information of the noise variance. The QRD-based BT-C method can outperform the SVD-based method by $1.8 \mathrm{~dB}$ at the BER of $10^{-4}$. We also observe that the QRD-based BT-C method provides near-optimum performance. These results indicate that the $[\mathbf{R}]$-value spread can effectively be reduced with the QRD-based BT-C method. The performance of the CLLL depends on the parameter $\delta$. As mentioned, if $\delta$ is smaller (close to 0.5 ), then the computational complexity will be lower. However, the performance will be poorer. As the value of $\delta$ we use is close to 1 , the columns of the channel matrix will approximately be orthogonal after the transformation. Thus, the number of channel columns to be permutated can be chosen as a smaller value for the reduction of the computational complexity. Here, we let $\bar{M}=2$. In other words, only two permutation patterns are considered. As we can see, the performance of the QRD-based BT-E method is almost the same as that of the QRD-based BT-C method. Note that the 


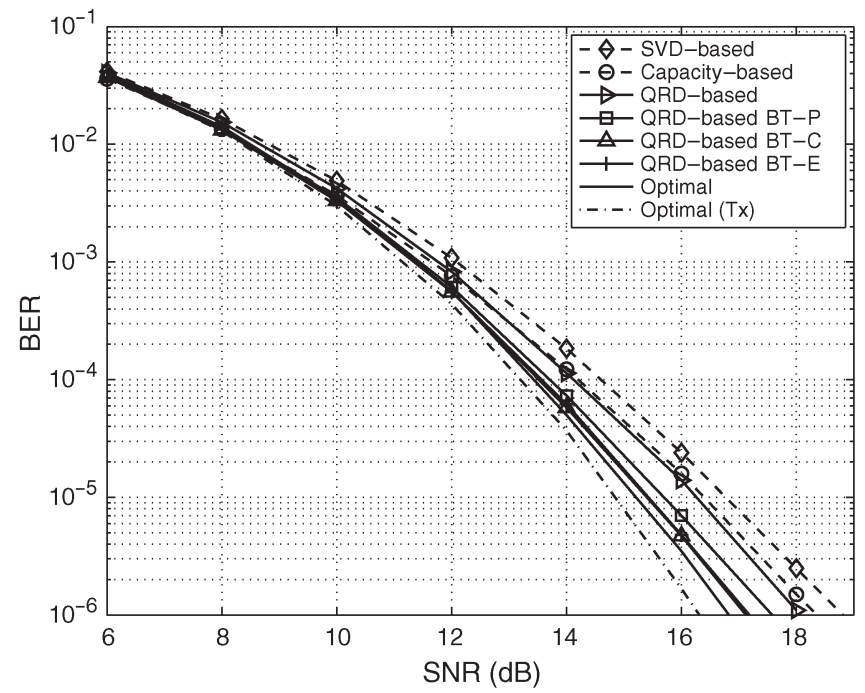

Fig. 6. BER performance comparison for receive antenna selection $\left(N_{t}=3\right.$, $N_{r}=6$, and $M=3$ ).

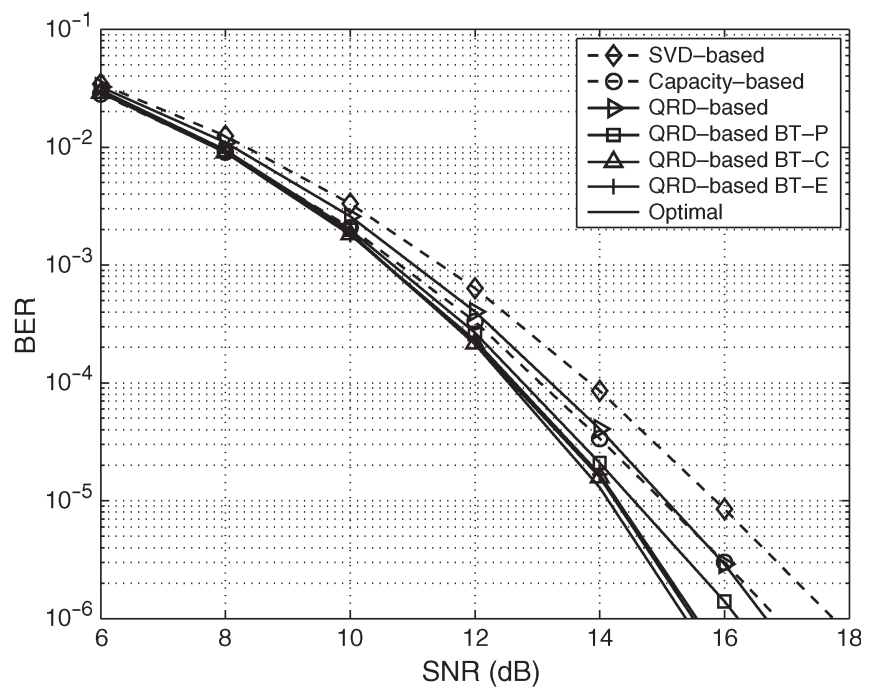

Fig. 7. BER performance comparison for joint transmit/receive antenna selection $\left(N_{t}=5, N_{r}=4\right.$, and $\left.M=3\right)$.

computational complexity of the QRD-based BT-E method is much lower.

Fig. 6 shows the BER comparison for receive antenna selection. We assume that $N_{t}=3, N_{r}=6$, and $M=3$. The results show that the proposed QRD-based BT-C method can also achieve near-optimum performance. As for other methods, a similar behavior can be observed. Note that we also let the number of candidate channel matrices equal to that in Fig. 5. This allows us to verify the analysis shown in Section V-A. From Fig. 6, we can see that receive antenna selection indeed suffers from performance loss compared to transmit antenna selection, which is consistent with our analysis. Furthermore, another observation is that the performance gaps between various methods are slightly reduced in Fig. 6. This result can also be attributed to the correlation between candidate channel matrices.

Fig. 7 compares the performance of various selection schemes for joint transmit/receive antenna selection. Similarly, we let $N_{t}=5, N_{r}=4$, and $M=3$ such that the number of the

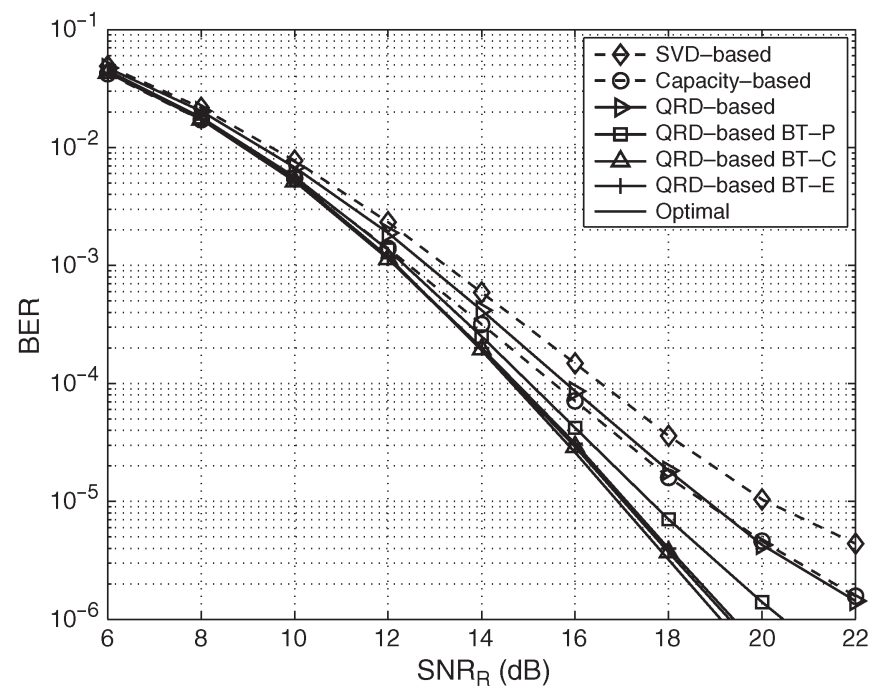

Fig. 8. BER performance comparison for antenna selection in a two-hop MIMO relay system $\left(N_{t}=4, N_{\text {re }}=4, N_{r}=4\right.$, and $\left.M=3\right)$.

total antennas remains the same. Distributing the extra antennas at both the transmitter and the receiver sides, we can have $\left(\begin{array}{l}5 \\ 3\end{array}\right) \times\left(\begin{array}{l}4 \\ 3\end{array}\right)$ candidate channel matrices to choose from. Note that the receive antenna selection is involved, indicating that its performance may be worse than the pure transmit antenna selection with the same total number of antennas. However, the number of the candidate channel matrices becomes larger in this case, and the selection performance can then be enhanced. Overall, the performance is improved in all the methods.

Next, we consider the performance comparison for a two-hop AF MIMO relay system. Fig. 8 shows the simulation results. In the figure, the performance is evaluated as a function of the average SNR per antenna at the relay, which is denoted as $S_{N R}$. In addition, the average SNR per antenna at the destination, which is denoted by $\mathrm{SNR}_{\mathrm{D}}$, is assumed to be $25 \mathrm{~dB}$. Here, we let $N_{t}=4, N_{r}=4, N_{\mathrm{re}}=4$, and $M=3$. In this case, we require two bits for sending the optimum indices of antenna subsets back to the source and the relay node, respectively. With the system model described in Section V-B, the relay node can receive and retransmit signal with different antenna subsets, which means we have $\left(\begin{array}{l}4 \\ 3\end{array}\right) \times\left(\begin{array}{l}4 \\ 3\end{array}\right) \times\left(\begin{array}{l}4 \\ 3\end{array}\right) \times\left(\begin{array}{l}4 \\ 3\end{array}\right)$ candidate channel matrices to be evaluated, and the computational complexity can be very high. Thus, we can consider a simplified scheme where the same antenna subset is used for signal reception and retransmission at the relay. With this simplification, the number of candidate channel matrices is reduced to 64 . Similar to the previous results, the proposed QRD-based BT-C method can still achieve near-optimum performance. In this scenario, it outperforms the SVD-based method by $2 \mathrm{~dB}$ at the BER of $10^{-4}$. Note that all the schemes will exhibit an error floor when $\mathrm{SNR}_{\mathrm{R}}$ is close to $\mathrm{SNR}_{\mathrm{D}}$. The reason is that as $\sigma_{\mathrm{SR}}^{2}$ is small, the system performance is dominated by $\sigma_{\mathrm{RD}}^{2}$.

Finally, we provide the BER performance comparison when the ML detection is implemented with the SDA. Transmit antenna selection is considered, and the result is shown in Fig. 9. The search radius $\gamma$ is determined according to $\gamma^{2}=\kappa \times$ $\operatorname{det}\left(\mathbf{H}_{p} \mathbf{H}_{p}^{H}\right)^{(1 / 2 M)}$ [26]. We set $\kappa=5$ so that the SDA can provide good performance and at the same time its computational 


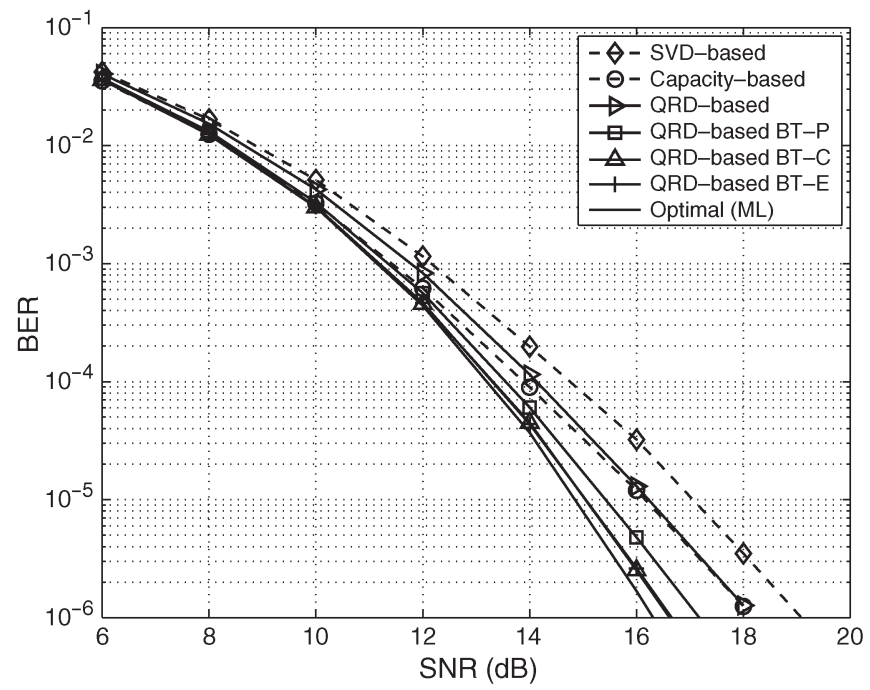

Fig. 9. BER performance comparison for transmit antenna selection with SDA $\left(N_{t}=6, N_{r}=3\right.$, and $\left.M=3\right)$.

complexity remains reasonably low. From Fig. 9, we can observe that the behavior of each scheme is very similar to that in Fig. 5. The performance of the QRD-based BT-C scheme with the SDA is still comparable with that of the optimum method.

\section{CONCLUSION}

In this paper, we have considered antenna selection methods in spatially multiplexed MIMO systems. First, we proposed a QRD-based transmit antenna selection method for ML receivers. The theoretical analysis and simulation results indicated that the QRD-based method not only outperforms the conventional SVD-based method but has a lower computational complexity as well. To further improve the performance, a basis transformation method was then proposed. Two transformation matrices were proposed to conduct the basis transformation, i.e., the permutation matrix and the LR matrix. The cascade of the permutation and LR matrices can also serve as another transformation matrix. We have shown that the basis transformation method can effectively tighten the original QRDbased lower bound. Efficient methods were also developed for the reduction of the computational complexity in the proposed selection schemes. We have also considered the applications of the proposed methods in other selection scenarios. Simulation results showed that the proposed basis transformation method can provide near-optimum performance.

\section{APPENDIX A}

\section{Singular Value Decomposition-Based Lower BOUND With THE TRANSFORMED SyMBOL VECTOR}

In what follows, we will show that the SVD-based lower bound can still be used when a transformation is conducted on $\mathrm{x}$. Starting from (16), we can rewrite the free distance as

$$
d_{\text {free }}=\min _{\overline{\mathbf{x}}, \overline{\mathbf{x}}^{\prime} \in \overline{\mathcal{X}}^{M}, \overline{\mathbf{x}} \neq \overline{\mathbf{x}}^{\prime}} \frac{\left\|\overline{\mathbf{H}}_{p}\left(\overline{\mathbf{x}}-\overline{\mathbf{x}}^{\prime}\right)\right\|^{2}}{\left\|\overline{\mathbf{x}}-\overline{\mathbf{x}}^{\prime}\right\|^{2}}\left\|\overline{\mathbf{x}}-\overline{\mathbf{x}}^{\prime}\right\|^{2} .
$$

Using the Rayleigh-Ritz theorem, we can have the following result:

$$
\frac{\left\|\overline{\mathbf{H}}_{p}\left(\overline{\mathbf{x}}-\overline{\mathbf{x}}^{\prime}\right)\right\|^{2}}{\left\|\overline{\mathbf{x}}-\overline{\mathbf{x}}^{\prime}\right\|^{2}} \geq \min _{\overline{\mathbf{x}} \neq \overline{\mathbf{x}}^{\prime}} \frac{\left\|\overline{\mathbf{H}}_{p}\left(\overline{\mathbf{x}}-\overline{\mathbf{x}}^{\prime}\right)\right\|^{2}}{\left\|\overline{\mathbf{x}}-\overline{\mathbf{x}}^{\prime}\right\|^{2}}=\bar{\lambda}_{M}^{2}
$$

where $\bar{\lambda}_{M}$ is the minimum singular value of $\overline{\mathbf{H}}_{p}$. Consequently, we can have the modified SVD-based lower bound expressed as

$$
d_{\text {free }} \geq \bar{\lambda}_{M}^{2} d_{\min }\left(\overline{\mathcal{X}}^{M}\right)
$$

where $d_{\min }\left(\overline{\mathcal{X}}^{M}\right)$ is defined as (5). From (44), it can be seen that the SVD-based lower bound is still valid though the entries of $\overline{\mathbf{x}}$ are correlated. However, $d_{\min }\left(\overline{\mathcal{X}}^{M}\right)$ is no longer straightforward to obtain. Finding $d_{\min }\left(\overline{\mathcal{X}}^{M}\right)$ may require an exhaustive search algorithm.

\section{APPENDIX B \\ EVALUATION OF $d_{\min }\left(\mathcal{X}_{\mathrm{LR}}^{1}\right)$}

For ease of description, we assume that the symbol vector is scaled and shifted such that the symbols before and after the transformation are located on the same lattice. As defined, for an $M \times M$ MIMO system, the LR-transformed symbol vector will be $\mathbf{x}_{\mathrm{LR}}=\mathbf{P}^{-1} \mathbf{x}$. Let $\mathbf{x}_{\mathrm{LR}}=\left[x_{\mathrm{LR}, 1}, x_{\mathrm{LR}, 2}, \ldots, x_{\mathrm{LR}, M}\right]^{T}$. Denote the $(n, k)$ th entry of $\mathbf{P}^{-1}$ as $P_{n, k}^{I}$. Then, the $n$th entry of $\mathrm{x}_{\mathrm{LR}}$ can be obtained by

$$
x_{\mathrm{LR}, n}=\sum_{k=1}^{M} P_{n, k}^{I} x_{k} .
$$

Note that $P_{n, k}^{I}$ is a complex integer, and $x_{\mathrm{LR}, n}$ is a combination of $x_{1}, x_{2}, \ldots$, and $x_{M}$. If $x_{k}$ has $S$ constellation points, then $x_{\mathrm{LR}, n}$ may have $S^{M}$ constellation points. As we can see, the number of the constellation points for $x_{\mathrm{LR}, n}$ can greatly be increased. As defined, $d_{\min }\left(\mathcal{X}_{\mathrm{LR}, n}\right)=\min \left|x_{n}-x_{n}^{\prime}\right|^{2}$. Note that $d_{\min }\left(\mathcal{X}_{\mathrm{LR}, n}\right)$ is changed only when no constellation points are allocated in the neighbor. In addition, $d_{\min }\left(\mathcal{X}_{\mathrm{LR}}^{1}\right)$ is changed only when all $d_{\min }\left(\mathcal{X}_{\mathrm{LR}, n}\right)$ 's are changed.

We now use an example to illustrate this property. Consider a $2 \times 2$ system with QPSK modulation for each transmit antenna. Let an LR matrix $\mathbf{P}$ be given by

$$
\mathbf{P}=\left[\begin{array}{cc}
2-1 j & 4+1 j \\
1 & 1+1 j
\end{array}\right]
$$

Assume that the minimum distance of the original symbol constellation is $g$. That is

$$
d_{\min }\left(\mathcal{X}_{1}\right)=d_{\min }\left(\mathcal{X}_{2}\right)=d_{\min }\left(\mathcal{X}^{M}\right)=g .
$$

Note that LR makes the transformed vectors located on the original constellation lattice. Therefore, we can have the result that $d_{\min }\left(\mathcal{X}_{\mathrm{LR}, 1}\right) \geq g$ and $d_{\min }\left(\mathcal{X}_{\mathrm{LR}, 2}\right) \geq g$. Fig. 10 shows the constellations before and after the transformation. It can be seen that $d_{\min }\left(\mathcal{X}_{\mathrm{LR}, 1}\right)>g$ while $d_{\min }\left(\mathcal{X}_{\mathrm{LR}, 2}\right)=g$. As defined, we have

$$
d_{\text {min }}\left(\mathcal{X}_{\mathrm{LR}}^{1}\right)=\min \left\{d_{\min }\left(\mathcal{X}_{\mathrm{LR}, 1}\right), d_{\min }\left(\mathcal{X}_{\mathrm{LR}, 2}\right)\right\}=g .
$$

From (46), we can see that $d_{\min }\left(\mathcal{X}_{\mathrm{LR}}^{1}\right)=d_{\min }\left(\mathcal{X}^{M}\right)$ when at least one of $\left\{d_{\min }\left(\mathcal{X}_{\mathrm{LR}, 1}\right), d_{\min }\left(\mathcal{X}_{\mathrm{LR}, 2}\right)\right\}$ is equal to $g$. This 

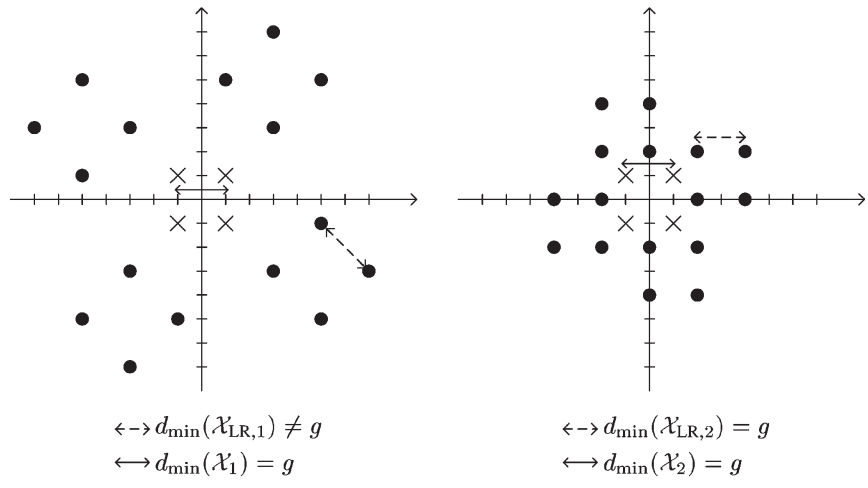

Fig. 10. Symbol constellations in which $d_{\min }\left(\mathcal{X}_{\mathrm{LR}}^{1}\right)=d_{\min }\left(\mathcal{X}^{M}\right)$ $(M=2, \times$ : original constellation, $\bullet$ : transformed constellation).

result clearly indicates that $d_{\min }\left(\mathcal{X}_{\mathrm{LR}}^{1}\right)$ is larger than $g$ only when all $d_{\min }\left(\mathcal{X}_{\mathrm{LR}, i}\right)$ 's are changed simultaneously. Fortunately, the case that all $d_{\min }\left(\mathcal{X}_{\mathrm{LR}, i}\right)$ 's are changed can be of very low probability when $M$ increases. From the simulations, we found that the probability of $d_{\min }\left(\mathcal{X}_{\mathrm{LR}}^{1}\right) \neq g$ is about $10^{-3}$ for $M=2$, and it can be lower than $10^{-6}$ when $M=3$. Thus, we can assume that the value of $d_{\min }\left(\mathcal{X}_{\mathrm{LR}}^{1}\right)$ is the same for all the candidate channel matrices.

\section{APPENDIX C \\ PROOF OF PROPOSITION 2}

In the CLLL algorithm shown in Table II, the reduction operations are performed on two neighbor columns sequentially, from the left to the right column pairs. Consider a candidate channel matrix $\mathbf{H}_{p}$ with its $\mathrm{QRD}$ expressed as $\mathbf{H}_{p}=\mathbf{Q R}$. For the $(m-1)$ th neighbor column pair of $\mathbf{R}$, the swap will occur when the condition that $\delta\left|R_{m-1, m-1}\right|^{2}>\left|R_{m, m}\right|^{2}+$ $\left|R_{m-1, m}\right|^{2}$ is met, where $m=2,3, \ldots, M$. Let $\Pi^{(m-1)}$ be a permutation matrix that swaps the $(m-1)$ th and $m$ th columns of $\mathbf{R}$. We can have $\mathbf{H}_{p} \boldsymbol{\Pi}^{(m-1)}=\mathbf{Q R} \boldsymbol{\Pi}^{(m-1)}=\mathbf{Q} \mathbf{R}^{\prime}$. Then, the Givens rotation matrix $\Theta$, which is expressed as

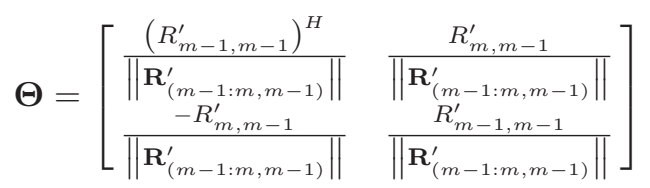

is applied so that $\mathbf{R}^{\prime}$ can be transformed into an upper triangular matrix denoted as $\mathbf{R}^{\prime \prime}$. Letting

$$
\mathbf{R}_{(m-1: m, m-1: M)}^{\prime \prime}=\boldsymbol{\Theta} \mathbf{R}_{(m-1: m, m-1: M)}^{\prime}
$$

we can have

$$
\begin{aligned}
& \left|R_{1,1}^{\prime \prime}\right|^{2}=\frac{\left(\left|R_{m-1, m}\right|^{2}+\left|R_{m, m}\right|^{2}\right)^{2}}{w} \\
& \left|R_{2,2}^{\prime \prime}\right|^{2}=\frac{\left|R_{m-1, m-1}\right|^{2}\left|R_{m, m}\right|^{2}}{w}
\end{aligned}
$$

where $w=\left|R_{m-1, m}\right|^{2}+\left|R_{m, m}\right|^{2}$ is a real-valued number. Then

$$
\begin{aligned}
\left|R_{1,1}^{\prime \prime}\right|^{2} & =\frac{\left(\left|R_{m-1, m}\right|^{2}+\left|R_{m, m}\right|^{2}\right)^{2}}{w} \\
& =\left|R_{m-1, m}\right|^{2}+\left|R_{m, m}\right|^{2} \\
& \geq\left|R_{m, m}\right|^{2} .
\end{aligned}
$$

Notice that $\delta\left|R_{m-1, m-1}\right|^{2}>\left|R_{m, m}\right|^{2}+\left|R_{m-1, m}\right|^{2}$, which means $\left|R_{m-1, m-1}\right|^{2}>\left|R_{m, m}\right|^{2}+\left|R_{m-1, m}\right|^{2}$ since $0.5<\delta<1$. Hence, we have

$$
\left|R_{1,1}^{\prime \prime}\right|^{2}=\left|R_{m-1, m}\right|^{2}+\left|R_{m, m}\right|^{2}<\left|R_{m-1, m-1}\right|^{2} .
$$

From (49) and (50), we know

$$
\left|R_{m, m}\right|^{2} \leq\left|R_{1,1}^{\prime \prime}\right|^{2}<\left|R_{m-1, m-1}\right|^{2}
$$

Next, we will show that $\left|R_{2,2}^{\prime \prime}\right|^{2}$ also has a similar property as that in (51). First, $\left|R_{2,2}^{\prime \prime}\right|^{2}$ can be expressed as

$$
\begin{aligned}
\left|R_{2,2}^{\prime \prime}\right|^{2} & =\frac{\left|R_{m-1, m-1}\right|^{2}\left|R_{m, m}\right|^{2}}{w} \\
& =\frac{\left|R_{m-1, m-1}\right|^{2}\left|R_{m, m}\right|^{2}}{\left|R_{m-1, m}\right|^{2}+\left|R_{m, m}\right|^{2}} .
\end{aligned}
$$

Using the fact that $\left|R_{m, m}\right|^{2}+\left|R_{m-1, m}\right|^{2}<\left|R_{m-1, m-1}\right|^{2}$, we can have

$$
\left|R_{2,2}^{\prime \prime}\right|^{2}>\frac{\left|R_{m-1, m-1}\right|^{2}\left|R_{m, m}\right|^{2}}{\left|R_{m-1, m-1}\right|^{2}}=\left|R_{m, m}\right|^{2} .
$$

Second, it is obvious that $\left|R_{m-1, m}\right|^{2}+\left|R_{m, m}\right|^{2} \geq\left|R_{m, m}\right|^{2}$. Therefore

$$
\begin{aligned}
\left|R_{2,2}^{\prime \prime}\right|^{2} & =\frac{\left|R_{m-1, m-1}\right|^{2}\left|R_{m, m}\right|^{2}}{\left|R_{m-1, m}\right|^{2}+\left|R_{m, m}\right|^{2}} \\
& \leq \frac{\left|R_{m-1, m-1}\right|^{2}\left|R_{m, m}\right|^{2}}{\left|R_{m, m}\right|^{2}} \\
& =\left|R_{m-1, m-1}\right|^{2} .
\end{aligned}
$$

Combining (53) with (54), we have

$$
\left|R_{m, m}\right|^{2}<\left|R_{2,2}^{\prime \prime}\right|^{2} \leq\left|R_{m-1, m-1}\right|^{2}
$$

Using (51) and (55), we can see that when the swap operation occurs at the $(m-1)$ th column pair

$$
\begin{aligned}
\left|R_{m, m}\right|^{2} & \leq \min \left\{\left|R_{1,1}^{\prime \prime}\right|^{2},\left|R_{2,2}^{\prime \prime}\right|^{2}\right\} \\
& \leq \max \left\{\left|R_{1,1}^{\prime \prime}\right|^{2},\left|R_{2,2}^{\prime \prime}\right|^{2}\right\} \\
& \leq\left|R_{m-1, m-1}\right|^{2} .
\end{aligned}
$$

Then, we consider the condition for $\delta\left|R_{m-1, m-1}\right|^{2} \leq$ $\left|R_{m, m}\right|^{2}+\left|R_{m-1, m}\right|^{2}$, where the swap operation is not conducted. Obviously, $\left|R_{m, m}\right|^{2}=\left|R_{2,2}^{\prime \prime}\right|^{2}$, and $\left|R_{m-1, m-1}\right|^{2}=$ $\left|R_{1,1}^{\prime \prime}\right|^{2}$. Note that, in this case, we cannot determine if $\left|R_{m-1, m-1}\right|^{2}$ or $\left|R_{m, m}\right|^{2}$ is larger. Taking this case into consideration, we can have the following inequality:

$$
\begin{aligned}
& \min \left\{\left|R_{m-1, m-1}\right|^{2},\left|R_{m, m}\right|^{2}\right\} \\
& \quad \leq \min \left\{\left|R_{1,1}^{\prime \prime}\right|^{2},\left|R_{2,2}^{\prime \prime}\right|^{2}\right\} \\
& \leq \max \left\{\left|R_{1,1}^{\prime \prime}\right|^{2},\left|R_{2,2}^{\prime \prime}\right|^{2}\right\} \\
& \quad \leq \max \left\{\left|R_{m-1, m-1}\right|^{2},\left|R_{m, m}\right|^{2}\right\} .
\end{aligned}
$$

From the results of (57)-(59), we observe that the swap operation in the CLLL algorithm can enlarge the value of 
$\min \left\{\left|R_{m-1, m-1}\right|^{2},\left|R_{m, m}\right|^{2}\right\}$. Let $[\mathbf{R}]_{\min }^{(m-1)}$ denote the minimum diagonal entry of $\mathbf{R}^{\prime \prime}$ when the CLLL algorithm has processed the $(m-1)$ th neighbor column pair. Note that $[\mathbf{R}]_{\min }^{(m-1)}$ is not necessarily identical to $\min \left\{\left|R_{m-1, m-1}\right|\right.$, $\left.\left|R_{m, m}\right|\right\}$. In addition, let $[\mathbf{R}]_{\min }^{(0)}=[\mathbf{R}]_{\min }$. We can also see that $[\mathbf{R}]_{\min }^{(M-1)} \geq[\mathbf{R}]_{\min }^{(0)}$ when the CLLL algorithm has completed its processing. Since $[\mathbf{R}]_{\min }^{(M-1)}=\left[\mathbf{R}_{\mathrm{LR}}\right]_{\min }$, we can conclude that $\left[\mathbf{R}_{\mathrm{LR}}\right]_{\min } \geq[\mathbf{R}]_{\min }$.

\section{REFERENCES}

[1] G. J. Foschini, "Layered space-time architecture for wireless communication in a fading environment when using multiple antennas," Bell Labs Tech. J., vol. 1, no. 2, pp. 41-59, 1996.

[2] L. Zheng and D. N. C. Tse, "Diversity and multiplexing: A fundamental tradeoff in multiple antenna channels," IEEE Trans. Inf. Theory, vol. 49, no. 5, pp. 1073-1096, May 2003.

[3] S. Sanayei and A. Nosratinia, "Capacity of MIMO channels with antenna selection," IEEE Trans. Inf. Theory, vol. 53, no. 11, pp. 4356-4362, Nov. 2007.

[4] R. W. Heath, S. Sandhu, and A. Paulraj, "Antenna selection for spatial multiplexing systems with linear receivers," IEEE Commun. Lett., vol. 5, no. 4, pp. 142-144, Apr. 2001.

[5] C. Mun, "Transmit-antenna selection for spatial multiplexing with ordered successive interference cancellation," IEEE Trans. Commun., vol. 54, no. 3, pp. 423-429, Mar. 2006.

[6] F. Kharrat-Kammoun, S. Fontenelle, S. Rouquette, and J. Boutros, "Antenna selection for MIMO systems based on an accurate approximation of QAM error probability," in Proc. IEEE Veh. Technol. Conf., Stockholm, Sweden, May 2005, pp. 206-210.

[7] R. W. Heath, Jr. and A. Paulraj, "Antenna selection for spatial multiplexing systems based on minimum error rate," in Proc. IEEE Int. Conf. Commun., Helsinki, Finland, Jun. 2001, vol. 7, pp. 2276-2280.

[8] H. Yao and G. W. Wornell, "Lattice-reduction-aided detectors for MIMO communication systems," in Proc. IEEE Global Telecommun. Conf., Taipei, Taiwan, Nov. 2002, pp. 424-428.

[9] D. Wübben, R. Böhnke, V. Kühn, and K.-D. Kammeyer, "Nearmaximum-likelihood detection of MIMO systems using MMSE-based lattice-reduction," in Proc. IEEE Int. Conf. Commun., Paris, France, Jun. 2004, pp. 798-802.

[10] I. Berenguer and X. Wang, "MIMO antenna selection with latticereduction-aided linear receivers," IEEE Trans. Veh. Technol., vol. 53, no. 5, pp. 1289-1302, Sep. 2004

[11] A. Gorokhov, D. A. Gore, and A. J. Paulraj, "Receive antenna selection for MIMO spatial multiplexing: Theory and algorithms," IEEE Trans. Signal Process., vol. 51, no. 11, pp. 2796-2807, Nov. 2003.

[12] K. T. Phan and C. Tellambura, "Receive antenna selection based on unionbound minimization using convex optimization," IEEE Signal Process. Lett., vol. 14, no. 9, pp. 609-612, Sep. 2007.

[13] A. F. Molisch, M. Z. Win, Y.-S. Choi, and J. H. Winters, "Capacity of MIMO systems with antenna selection," IEEE Trans. Wireless Commun., vol. 4, no. 4, pp. 1759-1772, Jul. 2005.

[14] T. Gucluoglu and T. M. Duman, "Performance analysis of transmit and receive antenna selection over flat fading channels," IEEE Trans. Wireless Commun., vol. 7, no. 8, pp. 3056-3065, Aug. 2008.
[15] W. Guan and H. Luo, "Joint MMSE transceiver design in non-regenerative MIMO relay systems," IEEE Commun. Lett., vol. 12, no. 7, pp. 517-519, Jul. 2008.

[16] X. Tang and Y. Hua, "Optimal design of non-regenerative MIMO wireless relays," IEEE Trans. Wireless Commun., vol. 6, no. 4, pp. 1398-1407, Apr. 2007.

[17] J.-K. Zhang, A. Kavcic, and K. M. Wong, "Equal-diagonal QR decomposition and its application to precoder design for successive-cancellation detection," IEEE Trans. Inf. Theory, vol. 51, no. 1, pp. 154-172, Jan. 2005.

[18] T. Guess, "Optimal sequences for CDMA with decision-feedback receivers," IEEE Trans. Inf. Theory, vol. 49, no. 4, pp. 886-900, Apr. 2003.

[19] R. A. Horn and C. R. Johnson, Matrix Analysis. New York: Cambridge Univ. Press, 1985.

[20] A. W. Marshall and I. Olkin, Inequalities: Theory of Majorization and its Applications. New York: Academic, 1991.

[21] G. Golub and C. F. Van Loan, Matrix Computations, 3rd ed. Baltimore, MD: Johns Hopkins Univ. Press, 1996.

[22] N. Balakrishnan and A. C. Cohen, Order Statistics and Inference Estimation Methods. New York: Academic, 1991.

[23] W. Zhao and G. B. Giannakis, "Reduced complexity closest point decoding algorithms for random lattices," IEEE Trans. Wireless Commun., vol. 5, no. 1, pp. 101-111, Jan 2006.

[24] K. J. Kim, M.-O. Pun, and R. A. Iltis, "QRD-based precoded MIMOOFDM systems with reduced feedback," in Proc. IEEE Int. Conf. Commun., Beijing, China, May 2008, pp. 708-712.

[25] X. Ma and W. Zhang, "Performance analysis for MIMO systems with lattice-reduction aided linear equalization," IEEE Trans. Commun., vol. 56, no. 2, pp. 309-318, Feb. 2008.

[26] J.-Y. Wu, W.-R. Wu, and N.-C. Lien, "Low-complexity MIMO detection using a list projection technique," in Proc. IEEE Int. Conf. Signal Process. Commun. Syst., Gold Coast, Australia, Dec. 2008, pp. 1-5.

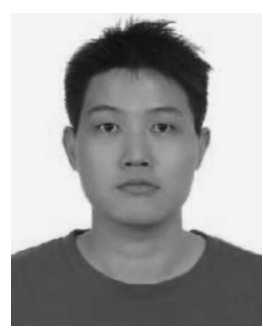

Chun-Tao Lin (S'11) received the B.S. degree in electrical engineering in 2002 from the National Chung Cheng University, Chiayi, Taiwan, and the M.S. degree in communications engineering in 2004 from the National Chiao Tung University, Hsinchu, Taiwan, where he is currently working toward the $\mathrm{Ph} . \mathrm{D}$. degree with the Institute of Communications Engineering.

His research interests include digital communication and MIMO signal processing.

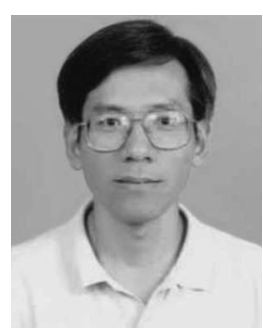

Wen-Rong Wu (M'89) received the B.S. degree in mechanical engineering from Tatung Institute of Technology, Taipei, Taiwan, in 1980 and the M.S. degree in mechanical and electrical engineering and the Ph.D. degree in electrical engineering from the State University of New York at Buffalo in 1985, 1986, and 1989, respectively.

Since August 1989, he has been a Faculty Member with the Department of Communication Engineering, National Chiao Tung University, Hsinchu, Taiwan. His research interests include statistical signal processing and digital communication. 